Review

\title{
Lie Symmetries of Differential Equations: Classical Results and Recent Contributions
}

\author{
Francesco Oliveri \\ Department of Mathematics, University of Messina, Contrada Papardo, Viale Ferdinando Stagno \\ d'Alcontres 31, I-98166 Messina, Italy; Tel.: +39-090-6765065; Fax: +39-090-393502; \\ E-Mail: oliveri@mat520.unime.it
}

Received: 2 January 2010 / Accepted: 30 March 2010 / Published: 8 April 2010

\begin{abstract}
Lie symmetry analysis of differential equations provides a powerful and fundamental framework to the exploitation of systematic procedures leading to the integration by quadrature (or at least to lowering the order) of ordinary differential equations, to the determination of invariant solutions of initial and boundary value problems, to the derivation of conservation laws, to the construction of links between different differential equations that turn out to be equivalent. This paper reviews some well known results of Lie group analysis, as well as some recent contributions concerned with the transformation of differential equations to equivalent forms useful to investigate applied problems.
\end{abstract}

Keywords: lie point symmetries; invariance of differential equations; invertible mappings between differential equations

Classification: MSC 22E70, 34A05, 35A30, 58J70, 58J72.

\section{Introduction}

Symmetry (joined to simplicity) has been, is, and probably will continue to be, an elegant and useful tool in the formulation and exploitation of the laws of nature. The request of symmetry accounts for the regularities of the laws that are independent of some inessential circumstances. For instance, the reproducibility of experiments in different places at different times relies on the invariance of the laws 
of nature under space translation and rotation (homogeneity and isotropy of space), and time translation (homogeneity of time). Without such regularities physical events probably would remain out of our knowledge, and the formulation of the laws themselves would be impossible.

Figure 1. Sophus Lie (Nordfjordeid, 17 December 1842 - Christiania, 18 February 1899).

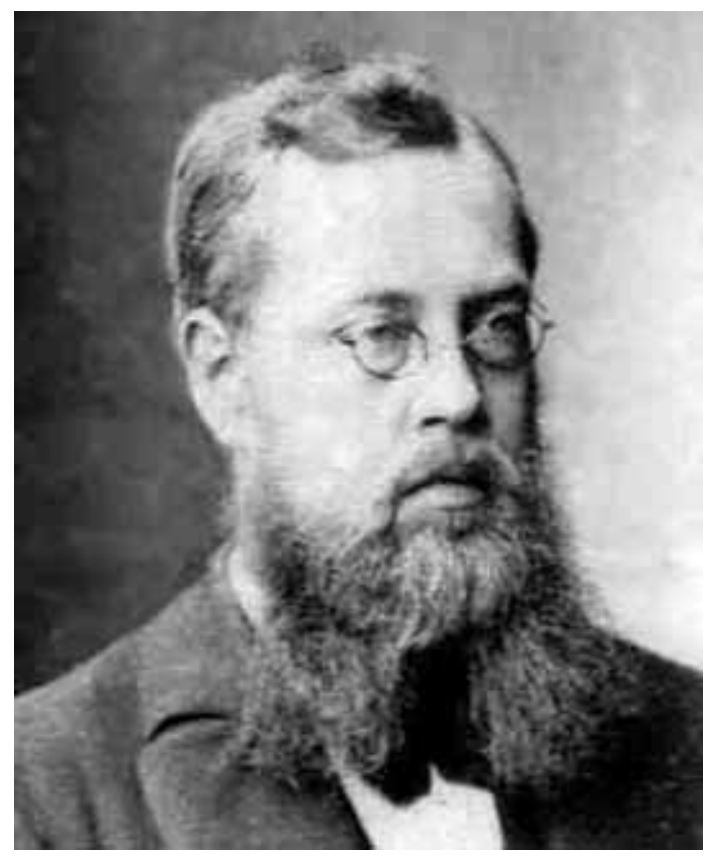

An important implication of symmetry in physics and in mathematics is the existence of conservation laws. This connection has been noticed in 1918, when Emmy Noether [1] proved her famous theorem relating continuous symmetries and conservation laws.

The concept of symmetry fascinated through the centuries many artists and scientists, from the ancient Greeks to Kepler, in his efforts for determining the orbits of the planets, to Newton, who embodied in the laws of mechanics - as a symmetry principle - the equivalence of motion in different inertial frames (Galilean invariance), to Einstein, who generalized the Galileo's principle of relativity from mechanics to all the laws of physics.

In the nineteenth century a great advance arose when the Norwegian mathematician Sophus Lie (Figure 1) began to investigate the continuous groups of transformations leaving differential equations invariant, creating what is now called the symmetry analysis of differential equations. The original Lie's aim was that of setting a general theory for the integration of ordinary differential equations similar to that developed by E. Galois e N. Abel for algebraic equations (see [2] for a detailed historical analysis).

Thus, symmetry analysis of differential equations was developed and applied by Sophus Lie during the period 1872-1899 [3,4]. This theory enables to derive solutions of differential equations in a completely algorithmic way without appealing to special lucky guesses. Sophus Lie pointed out the main problem he faced with his theory in a foreword to his lecture Differentialgleichungen given in Leipzig (1891):

The older examinations on ordinary differential equations as found in standard books are not systematic. The writers developed special integration theories for homogeneous differential 
equations, for linear differential equations, and other special integrable forms of differential equations. However, the mathematicians did not realize that these special theories are all contained in the term infinitesimal transformations, which is closely connected with the term of a one parametric group.

Despite this feature, the Lie's approach to differential equations was not exploited for half a century and only the abstract theory of Lie groups grew (the term Lie group has been coined in 1928 by Hermann Weyl). It was in the Forties of last century, with the work of G. Birkhoff [5] and I. Sedov [6] on dimensional analysis, that the theory gave relevant results in concrete applied problems. Further, it was the Russian school with L. V. Ovsiannikov [7] that since 1960 began to exploit systematically the methods of symmetry analysis of differential equations in the explicit construction of solutions of any sort of problems, even complicated, of mathematical physics. During the last few decades, there has been a revival of interest in Lie's theory and significant progress has been made from either a theoretical or an applied point of view. Many monographies and textbooks are now available [7-19], and an increasing number of research papers is currently published.

Lie's theory is powerful, versatile, and fundamental to the development of systematic procedures leading to the integration by quadrature (or at least to lowering the order) of ordinary differential equations, to the determination of invariant solutions of initial and boundary value problems, to the derivation of conserved quantities, or to the construction of relations between different differential equations that turn out to be equivalent.

The application of Lie's theory to differential equations is completely algorithmic; however, it usually involves a lot of cumbersome and tedious calculations. For instance, in looking for symmetries of a system of partial differential equations, it is not uncommon to have to handle hundreds of equations to find a single solution. Today, we have access to powerful Computer Algebra Systems (CAS) like Maple ${ }^{\circledR}$ and Mathematica ${ }^{\circledR}$ (commercial), Maxima and Reduce (open source), and the needed algebraic manipulations can be rapidly done automatically [15,20-24]. In fact, many specific packages for performing symmetry analysis of differential equations are currently available in the literature.

Lie's classical theory is a source for various generalizations. Among these generalizations there are the non-classical method first proposed by Bluman and Cole [25] and now part of the more general method of differential constraints [26-28], the introduction of approximate symmetries [29,30], generalized symmetries [10], which in turn generalize contact symmetries introduced by Lie's himself, equivalence transformations [7,18,31], and nonlocal symmetries [10,11,32], to quote a few.

The key idea of Lie's theory of symmetry analysis of differential equations relies on the invariance of the latter under a transformation of independent and dependent variables. This transformation forms a local group of point transformations establishing a diffeomorphism on the space of independent and dependent variables, mapping solutions of the equations to other solutions. Any transformation of the independent and dependent variables in turn induces a transformation of the derivatives. 


\section{Basic Theory of Lie Groups of Transformations}

In this Section and in the following one, in order to keep the paper self-contained, let us briefly sketch some basic elements of Lie group analysis of differential equations; a more detailed exposition can be found in [7-19].

Definition 1 (Groups of transformations) Let us consider a domain $D \subseteq \mathbb{R}^{N}$ and a subset $S \subseteq \mathbb{R}$. The set of transformations

$$
\mathbf{z}^{\star}=\mathbf{Z}(\mathbf{z} ; a), \quad \mathbf{Z}: D \times S \rightarrow D
$$

depending on the parameter a, forms a one-parameter group of transformations on $D$ if:

1. For each value of the parameter $a \in S$ the transformations are one-to-one onto $D$;

2. $S$ with the law of composition $\mu$ is a group with identity e;

3. $\mathbf{Z}(\mathbf{z} ; e)=\mathbf{z}, \forall \mathbf{z} \in D$;

4. $\mathbf{Z}(\mathbf{Z}(\mathbf{z} ; a) ; b)=\mathbf{Z}(\mathbf{z} ; \mu(a, b)), \forall \mathbf{z} \in D, \forall a, b \in S$.

Definition 2 (Lie group of transformations) The group of transformations (1) defines a one-parameter Lie group of transformations if in addition to satisfying the axioms of the previous definition:

1. a is a continuous parameter, i.e., $S$ is an interval in $\mathbb{R}$;

2. $\mathrm{Z}$ is $C^{\infty}$ with respect to $\mathrm{z}$ in $D$ and an analytic function of $a$ in $S$.

3. $\mu(a, b)$ is an analytic function of $a$ and $b, \forall a, b \in S$.

Due to the analyticity of the group operation $\mu$, it is always possible to reparametrize the Lie group in such a way the group operation becomes the ordinary sum in $\mathbb{R}$.

Expanding (1) in powers of $a$ around $a=0$, we get (in a neighbourhood of $a=0$ ):

$$
\mathbf{z}^{\star}=\mathbf{z}+\left.a \frac{\partial \mathbf{Z}(\mathbf{z} ; a)}{\partial a}\right|_{a=0}+\left.\frac{a^{2}}{2} \frac{\partial^{2} \mathbf{Z}(\mathbf{z} ; a)}{\partial a^{2}}\right|_{a=0}+\cdots=\mathbf{z}+\left.a \frac{\partial \mathbf{Z}(\mathbf{z} ; a)}{\partial a}\right|_{a=0}+O\left(a^{2}\right)
$$

By setting

$$
\boldsymbol{\zeta}(\mathbf{z})=\left.\frac{\partial \mathbf{Z}(\mathbf{z} ; a)}{\partial a}\right|_{a=0}
$$

the transformation

$$
\mathbf{z}^{\star}=\mathbf{z}+a \boldsymbol{\zeta}(\mathbf{z})
$$

defines the infinitesimal transformation of the Lie group of transformations, the components of $\boldsymbol{\zeta}(\mathbf{z})$ being the so called infinitesimals of (1).

Lie's First Fundamental Theorem ensures that the infinitesimal transformations contain the essential information for characterizing a one-parameter Lie group of transformations.

Theorem 1 (First Fundamental Theorem of Lie) The Lie group of transformations (1) is equivalent to the solution of the initial value problem for the system of first order differential equations

$$
\frac{d \mathbf{z}^{\star}}{d a}=\boldsymbol{\zeta}\left(\mathbf{z}^{\star}\right), \quad \mathbf{z}^{\star}(0)=\mathbf{z}
$$


The tangent vector field $\zeta(\mathbf{z})$ is written under the form of the first order differential operator (the symbol in Lie's notation)

$$
\Xi=\boldsymbol{\zeta}(\mathbf{z}) \cdot \boldsymbol{\nabla}=\zeta_{1}(\mathbf{z}) \frac{\partial}{\partial z_{1}}+\cdots+\zeta_{N}(\mathbf{z}) \frac{\partial}{\partial z_{N}}
$$

For any differentiable function $F(\mathbf{z})$ it is

$$
\Xi F=\boldsymbol{\zeta}(\mathbf{z}) \cdot \nabla F=\zeta_{1}(\mathbf{z}) \frac{\partial F}{\partial z_{1}}+\cdots+\zeta_{N}(\mathbf{z}) \frac{\partial F}{\partial z_{N}}
$$

and, in particular,

$$
\Xi \mathbf{z}=\zeta(\mathbf{z})
$$

A one-parameter Lie group of transformations, which by Theorem 1 is equivalent to its infinitesimal transformation, is also equivalent to its infinitesimal operator. The latter allows to represent the solution of Lie's equations in terms of a Taylor series (exponential map):

$$
\mathbf{z}^{\star}=\exp (a \Xi) \mathbf{z}=\mathbf{z}+a \Xi \mathbf{z}+\frac{a^{2}}{2} \Xi^{2} \mathbf{z}+\cdots=\sum_{k=0}^{\infty} \frac{a^{k}}{k !} \Xi^{k} \mathbf{z}
$$

where $\Xi^{k} \mathbf{z}=\Xi\left(\Xi^{k-1} \mathbf{z}\right), \Xi^{0} \mathbf{z}=\mathbf{z}$.

Now, we can introduce the concept of invariance of a function with respect to a Lie group of transformations, and prove the related invariance criterion.

An infinitely differentiable function $F(\mathbf{z})$ is said to be an invariant function (or, simply, an invariant) of the Lie group of transformations (1) if and only if for any group transformation (1) the condition

$$
F\left(\mathbf{z}^{\star}\right) \equiv F(\mathbf{z})
$$

holds true. The invariance of a function is characterized in a very simple way by means of the infinitesimal generator of the group, as the following theorem shows.

Theorem $2 F(\mathbf{z})$ is invariant under (1) if and only if

$$
\Xi F(\mathbf{z})=0
$$

It can be also defined the invariance of a surface of $\mathbb{R}^{N}$ with respect to a Lie group. A surface $F(\mathbf{z})=0$ is said to be an invariant surface with respect to the one-parameter Lie group (1) if $F\left(\mathbf{z}^{\star}\right)=0$ when $F(\mathbf{z})=0$. As a consequence of the theorem 2, the following theorem immediately follows.

Theorem 3 A surface $F(\mathbf{z})=0$ is invariant with respect to the group (1) if and only if

$$
\Xi F(\mathbf{z})=0 \quad \text { when } \quad F(\mathbf{z})=0
$$

For any Lie group there exists a set of privileged variables (canonical variables) in terms of which the Lie group (and the associated infinitesimal generator) expresses in its simplest form.

Given in $\mathbb{R}^{N}$ the one-parameter Lie group of transformations (1), let us suppose to make the change of variables defined by the one-to-one and $C^{1}$ transformation:

$$
\mathbf{y}=\mathbf{Y}(\mathbf{z})
$$


If we choose the function $\mathbf{Y}(\mathbf{z})$ such that the conditions

$$
\Xi Y_{i}(\mathbf{z})=0, \quad(i=1, \ldots, N-1), \quad \Xi Y_{N}(\mathbf{z})=1
$$

hold, then the infinitesimal generator expresses as

$$
\Xi=\frac{\partial}{\partial y_{N}}
$$

and the Lie group writes as

$$
y_{i}^{\star}=y_{i} \quad(i=1, \ldots, N-1), \quad y_{N}^{\star}=y_{N}+a
$$

A Lie group of transformations may depend as well on many parameters,

$$
\mathbf{z}^{\star}=\mathbf{Z}(\mathbf{z} ; \mathbf{a})
$$

where $\mathbf{a}=\left(a_{1}, a_{2}, \ldots, a_{r}\right) \in S \subseteq \mathbb{R}^{r}$. The infinitesimal matrix $\chi(\mathbf{z})$ is the $r \times N$ matrix with entries

$$
\zeta_{\alpha j}(\mathbf{z})=\left.\frac{\partial z_{j}^{\star}}{\partial a_{\alpha}}\right|_{\mathbf{a}=\mathbf{0}}=\left.\frac{\partial Z_{j}(\mathbf{x}, \mathbf{a})}{\partial a_{\alpha}}\right|_{\mathbf{a}=\mathbf{0}}
$$

$(\alpha=1, \ldots, r ; j=1, \ldots, N)$ may be constructed, and, for each parameter $a_{\alpha}$ of the $r$-parameter Lie group of transformations (15), the infinitesimal generator $\Xi_{\alpha}$,

$$
\Xi_{\alpha}=\sum_{j=1}^{N} \zeta_{\alpha j}(\mathbf{z}) \frac{\partial}{\partial z_{j}} \quad(\alpha=1 \ldots, r)
$$

is defined. The infinitesimal generator

$$
\Xi=\sum_{\alpha=1}^{r} \sigma_{\alpha} \Xi_{\alpha}=\sum_{j=1}^{N} \zeta_{j}(\mathbf{z}) \frac{\partial}{\partial z_{j}}, \quad \zeta_{j}(\mathbf{x})=\sum_{\alpha=1}^{r} \sigma_{\alpha} \zeta_{\alpha j}(\mathbf{z})
$$

where $\sigma_{1}, \ldots, \sigma_{r}$ are fixed real constants, in turn defines a one-parameter subgroup of an $r$-parameter Lie group of transformations.

\section{Lie Groups of Differential Equations}

In considering Lie groups of point transformations associated to a given differential equation $\mathcal{E}$ involving $n$ independent variables $\mathbf{x}=\left(x_{1}, \ldots, x_{n}\right) \in \mathbb{R}^{n}$, and $m$ dependent variables $\mathbf{u}=$ $\left(u^{1}, \ldots, u^{m}\right) \in \mathbb{R}^{m}$, let us write such a group of transformations in the form

$$
\mathbf{x}^{\star}=\mathbf{X}(\mathbf{x}, \mathbf{u} ; a), \quad \mathbf{u}^{\star}=\mathbf{U}(\mathbf{x}, \mathbf{u} ; a)
$$

acting on the space $\mathbb{R}^{n+m}$ of the variables $(\mathbf{x}, \mathbf{u})$. Also, let

$$
\mathbf{u}=\boldsymbol{\Theta}(\mathbf{x}) \equiv\left(\Theta^{1}(\mathbf{x}), \Theta^{2}(\mathbf{x}), \ldots, \Theta^{m}(\mathbf{x})\right)
$$

be a solution of the equation $\mathcal{E}$.

A Lie group of transformations of the form (18) admitted by $\mathcal{E}$ has the two equivalent properties: 
1. a transformation of the group maps any solution of $\mathcal{E}$ into another solution of $\mathcal{E}$;

2. a transformation of the group leaves $\mathcal{E}$ invariant, say, $\mathcal{E}$ reads the same in terms of the variables $(\mathbf{x}, \mathbf{u})$ and in terms of the transformed variables $\left(\mathbf{x}^{\star}, \mathbf{u}^{\star}\right)$.

The transformations (18) determine suitable transformations for the derivatives of the dependent variables $\mathbf{u}$ with respect to the independent variables $\mathbf{x}$.

Let $\mathbf{u}^{(1)}$ denote the set of all $m \cdot n$ first order partial derivatives of $\mathbf{u}$ with respect to $\mathbf{x}$,

$$
\mathbf{u}^{(1)} \equiv\left(\frac{\partial u^{1}}{\partial x_{1}}, \ldots, \frac{\partial u^{1}}{\partial x_{n}}, \ldots, \frac{\partial u^{m}}{\partial x_{1}}, \ldots, \frac{\partial u^{m}}{\partial x_{n}}\right)
$$

and, in general, let $\mathbf{u}^{(k)}$ denote the set of all $k$ th-order partial derivatives of $\mathbf{u}$ with respect to $\mathbf{x}$.

The transformations of the derivatives of the dependent variables lead to natural extensions (prolongations) of the one-parameter Lie group of transformations (18). While the one-parameter Lie group of transformations (18) acts on the space $(\mathbf{x}, \mathbf{u})$, the extended group acts on the space $\left(\mathbf{x}, \mathbf{u}, \mathbf{u}^{(1)}\right)$, and, more in general, on the jet space $\left(\mathbf{x}, \mathbf{u}, \mathbf{u}^{(1)}, \ldots, \mathbf{u}^{(k)}\right)$. Since all the information about a Lie group of transformations is contained in its infinitesimal generator, we need to compute its prolongations:

- the first order prolongation

$$
\Xi^{(1)}=\Xi+\sum_{A=1}^{m} \sum_{i=1}^{n} \eta_{[i]}^{A}\left(\mathbf{x}, \mathbf{u}, \mathbf{u}^{(1)}\right) \frac{\partial}{\partial u_{i}^{A}}, \quad u_{i}^{A}=\frac{\partial u^{A}}{\partial x_{i}}
$$

with

$$
\eta_{[i]}^{A}\left(\mathbf{x}, \mathbf{u}, \mathbf{u}^{(1)}\right)=\frac{D \eta^{A}}{D x_{i}}-\frac{D \xi_{j}}{D x_{i}} \frac{\partial u^{A}}{\partial x_{j}}
$$

- the general $k$ th-order prolongation recursively defined by

$$
\Xi^{(k)}=\Xi^{(k-1)}+\sum_{A=1}^{m} \sum_{i_{1}=1}^{n} \ldots \sum_{i_{k}=1}^{n} \eta_{\left[i_{1} \ldots i_{k}\right]}^{A} \frac{\partial}{\partial u_{i_{1} \ldots i_{k}}^{A}}, \quad u_{i_{1} \ldots i_{k}}^{A}=\frac{\partial^{k} u^{A}}{\partial x_{i_{1}} \ldots \partial x_{i_{k}}}
$$

where $u_{i_{1} \ldots i_{k}}^{A}=\frac{\partial^{k} u^{A}}{\partial x_{i_{1}} \ldots \partial x_{i_{k}}}$, and $\eta_{\left[i_{1} \ldots i_{k}\right]}^{A}$, defined recursively by the relation

$$
\eta_{\left[i_{1} \ldots i_{k}\right]}^{A}=\frac{D \eta_{\left[i_{1} \ldots i_{k-1}\right]}^{A}}{D x_{i_{k}}}-u_{i_{1} \ldots i_{k-1} j}^{A} \frac{D \xi_{j}}{D x_{i_{k}}}
$$

In (22) and (24) the Lie derivative

$$
\frac{D}{D x_{i}}=\frac{\partial}{\partial x_{i}}+\frac{\partial u^{A}}{\partial x_{i}} \frac{\partial}{\partial u^{A}}+\frac{\partial^{2} u^{A}}{\partial x_{i} \partial x_{j}} \frac{\partial}{\partial u_{j}^{A}}+\ldots
$$

has been introduced, and the Einstein convention of summation over repeated indices used.

The infinitesimals of the Lie group of transformations leaving a given system of differential equations invariant can be found by means of the straightforward algorithm that will be described below. 
Remarkably, the search of one-parameter Lie groups of transformations leaving differential equations invariant leads usually to obtain $r$-parameter Lie groups of transformations. Let

$$
\Delta\left(\mathbf{x}, \mathbf{u}, \mathbf{u}^{(1)}, \ldots, \mathbf{u}^{(k)}\right)=\mathbf{0}
$$

( $\Delta=\left(\Delta_{1}, \ldots, \Delta_{q}\right)$ ) be a system of $q$ differential equations of order $k$, with independent variables $\mathbf{x} \in \mathbb{R}^{n}$ and dependent variables $\mathbf{u} \in \mathbb{R}^{m}$. Suppose that the system (26) is written in normal form, i.e., it is solved with respect to some partial derivatives of order $k_{\nu}$ for $\nu=1, \ldots, q$ :

$$
\Delta_{\nu}\left(\mathbf{x}, \mathbf{u}, \mathbf{u}^{(1)}, \ldots, \mathbf{u}^{(k)}\right) \equiv u_{i_{1} \ldots i_{k_{\nu}}}^{A_{\nu}}-F_{\nu}\left(\mathbf{x}, \mathbf{u}, \mathbf{u}^{(1)}, \ldots, \mathbf{u}^{(k)}\right)=0
$$

The equations (27) can be considered as characterizing a submanifold in the $k$ th-order jet space, the latter having dimension equal to

$$
n+m \sum_{h=0}^{k}\left(\begin{array}{c}
n+h-1 \\
n-1
\end{array}\right)=n+m\left(\begin{array}{c}
n+k \\
k
\end{array}\right)
$$

It is said that the one-parameter Lie group of transformations (18) leaves the system (27) invariant (is admitted by (27)) if and only if its $k$ th prolongation leaves invariant the submanifold of the jet space defined by (27).

Theorem 3 allows to prove the following important theorem, which leads directly to the algorithm for the computation of the infinitesimals admitted by a given differential system.

\section{Theorem 4 (Infinitesimal Criterion for differential equations) Let}

$$
\Xi=\xi_{i}(\mathbf{x}, \mathbf{u}) \frac{\partial}{\partial x_{i}}+\eta^{A}(\mathbf{x}, \mathbf{u}) \frac{\partial}{\partial u^{A}}
$$

be the infinitesimal generator corresponding to (18), and $\Xi^{(k)}$ the kth extended infinitesimal generator. The group (18) is admitted by the system (27) if and only if

$$
\Xi^{(k)} \Delta\left(\mathbf{x}, \mathbf{u}, \mathbf{u}^{(1)}, \ldots, \mathbf{u}^{(k)}\right)=\mathbf{0} \quad \text { when } \quad \boldsymbol{\Delta}\left(\mathbf{x}, \mathbf{u}, \mathbf{u}^{(1)}, \ldots, \mathbf{u}^{(k)}\right)=\mathbf{0}
$$

If the differential system is in polynomial form in the derivatives, the invariance conditions (29) are polynomials in the components of $\left(\mathbf{u}^{(1)}, \ldots, \mathbf{u}^{(k)}\right)$, with coefficients expressed by linear combinations of the unknowns $\xi_{i}, \eta^{A}$ and their partial derivatives. After using (27) to eliminate the derivatives $u_{i_{1} \ldots i_{k_{\nu}}}^{A_{\nu}}$, the equations can be splitted with respect to the components of the remaining derivatives of $\mathbf{u}$ that can be arbitrarily varied. By equating to zero the coefficients of these partial derivatives, one obtains an overdetermined system of linear differential equations for the infinitesimals (the so called system of determining equations), whose integration leads to the infinitesimals of the group. The infinitesimals involve arbitrary constants (and in some cases arbitrary functions); therefore, we have de facto $r$-parameter Lie groups (infinite-parameter Lie groups when arbitrary functions are involved).

Throughout this paper we will deal mainly with Lie groups of transformations admitted by differential equations with infinitesimals depending on the independent and dependent variables only. These are called local Lie point symmetries. Symmetries in which the infinitesimals may depend also on first (respectively, higher) order derivatives of the dependent variables with respect to the independent 
variables are contact (respectively, generalized) symmetries, and symmetries with infinitesimals depending also on integrals of dependent variables are called nonlocal symmetries.

The infinitesimal generators of an $r$-parameter Lie group, being solutions of a linear system of partial differential equations, span an $r$-dimensional vector space; by introducing an operation of commutation between two infinitesimal generators,

$$
\left[\Xi_{\alpha}, \Xi_{\beta}\right]=\Xi_{\alpha} \Xi_{\beta}-\Xi_{\beta} \Xi_{\alpha}
$$

which is bilinear, antisymmetric, and satisfies the Jacobi identity, say

$$
\left[\left[\Xi_{\alpha}, \Xi_{\beta}\right], \Xi_{\gamma}\right]+\left[\left[\Xi_{\beta}, \Xi_{\gamma}\right], \Xi_{\alpha}\right]+\left[\left[\Xi_{\gamma}, \Xi_{\alpha}\right], \Xi_{\beta}\right]=0
$$

the vector space of infinitesimal generators gains the structure of a Lie algebra [33,34]. If $L$ is an $r$-dimensional vector space of infinitesimal generators closed under the operation of commutation, i.e., $L$ is an $r$-dimensional Lie algebra, and $\left\{\Xi_{1}, \ldots, \Xi_{r}\right\}$ is a basis, then it is

$$
\left[\Xi_{\alpha}, \Xi_{\beta}\right]=\sum_{\gamma=1}^{r} C_{\alpha \beta}^{\gamma} \Xi_{\gamma}
$$

with constant coefficients $C_{\alpha \beta}^{\gamma}$ known as structure constants; they transform like the components of a tensor under changes of bases.

In dealing with Lie algebras of transformations admitted by differential equations, two classes play a special role, the solvable and the Abelian Lie algebras. A Lie algebra is solvable if there exists a series

$$
L_{r} \supset L_{r-1} \supset \cdots \supset L_{1}
$$

of subalgebras of dimensions $r, r-1, \ldots, 1$ such that $L_{s}$ is an ideal in $L_{s+1}(s=1, \ldots, r-1)$.

A Lie algebra is Abelian if

$$
\left[\Xi_{\alpha}, \Xi_{\beta}\right]=0, \quad \alpha, \beta=1, \ldots r
$$

Finally, it is worth of being underlined that the commutator of two infinitesimal generators is invariant with respect to any invertible change of variables, and commutes with the operation of prolongation.

\section{Use of Lie Symmetries of Differential Equations}

The knowledge of Lie groups of transformations admitted by a given system of differential equations can be used

- to lower the order or eventually reduce the equation to quadrature, in the case of ordinary differential equations;

- to determine particular solutions, called invariant solutions, or generate new solutions, once a special solution is known, in the case of ordinary or partial differential equations. 


\subsection{Ordinary Differential Equations}

Lie showed that if a given ordinary differential equation admits a one-parameter Lie group of point transformations then the order of the equation can be lowered by one. Hence, the solution of the reduced equation and a quadrature provide the solution of the original ordinary differential equation. If a given ordinary differential equation admits an $r$-parameter Lie group of point transformations, the order of the equation can be lowered by $r$ if the corresponding Lie algebra is solvable. The lowering of the order of an ordinary differential equations can be obtained either by introducing the canonical variables or by constructing the differential invariants, the latter being invariants of the prolonged infinitesimal generators.

Bianchi [35] used solvable Lie groups (called integrable groups in earlier literature) to reduce the order of a system of first order ordinary differential equations. Olver [10] gave an existence theorem which shows that if an $n$ th-order ordinary differential equation admits an $r$-parameter solvable Lie group of transformations, then its general solution can be found by quadratures from the general solution of an $(n-r)$ th-order ordinary differential equation. Further, in [36] an iterative reduction algorithm has been given.

To illustrate briefly the method, let us consider a general first order ordinary differential equation

$$
u^{\prime}=\frac{d u}{d x}=f(x, u)
$$

and assume that it is left invariant by the Lie group of point transformations generated by

$$
\Xi=\xi(x, u) \frac{\partial}{\partial x}+\eta(x, u) \frac{\partial}{\partial u}
$$

By introducing the canonical variables $r(x, u)$ and $s(x, u)$,

$$
\Xi r=0, \quad \Xi s=1
$$

the infinitesimal generator (31) rewrites as

$$
\Xi=\frac{\partial}{\partial s}
$$

Furthermore, it results:

$$
\frac{d s}{d r}=\frac{s_{x}+s_{u} u^{\prime}}{r_{x}+r_{u} u^{\prime}}=\frac{s_{x}+s_{u} f(x, u)}{r_{x}+r_{u} f(x, u)}=F(r, s)
$$

But, since the latter equation must be invariant with respect to the Lie group (33), the function $F$ can not depend on $s$. Hence the original equation, written in terms of the canonical variables of the admitted Lie group, can be integrated by quadrature.

In general, for an $n$ th-order ordinary differential equations admitting an $r$-dimensional solvable Lie algebra of symmetries, the $n$ th-order ordinary differential equation is reduced to an $(n-r)$ th-order ordinary differential equation plus $r$ quadratures [11]; remarkably, one does not need to determine all the intermediate ordinary differential equations of decreasing order.

A good review of Lie group analysis of ordinary differential equations can be found in a paper [37] written for the 150th anniversary of Sophus Lie (see also [38] for a revisitation in terms of Pfaffian systems of the theory of integration through symmetries of $n$ th-order scalar ordinary differential equations). 


\subsection{Invariant Solutions of Partial Differential Equations}

The function $\mathbf{u}=\boldsymbol{\Theta}(\mathbf{x})$, with components $u^{A}=\Theta^{A}(\mathbf{x})(A=1,2, \ldots, m)$, is said to be an invariant solution of (26) if $u^{A}=\Theta^{A}(\mathbf{x})$ is an invariant surface of (18), and is a solution of (26), i.e., a solution is invariant if and only if:

$$
\begin{aligned}
& \Xi\left(u^{A}-\Theta^{A}(\mathbf{x})\right)=0 \quad \text { for } u^{A}=\Theta^{A}(\mathbf{x}) \quad(A=1, \ldots, m) \\
& \boldsymbol{\Delta}\left(\mathbf{x}, \mathbf{u}, \mathbf{u}^{(1)}, \ldots, \mathbf{u}^{(k)}\right)=\mathbf{0}
\end{aligned}
$$

The equations $(35)_{1}$, called invariant surface conditions, have the form

$$
\xi_{1}(\mathbf{x}, \mathbf{u}) \frac{\partial u^{A}}{\partial x_{1}}+\cdots+\xi_{n}(\mathbf{x}, \mathbf{u}) \frac{\partial u^{A}}{\partial x_{n}}=\eta^{A}(\mathbf{x}, \mathbf{u}) \quad(A=1, \ldots, m)
$$

and are solved by introducing the corresponding characteristic equations:

$$
\frac{d x_{1}}{\xi_{1}(\mathbf{x}, \mathbf{u})}=\cdots=\frac{d x_{n}}{\xi_{n}(\mathbf{x}, \mathbf{u})}=\frac{d u^{1}}{\eta^{1}(\mathbf{x}, \mathbf{u})}=\cdots=\frac{d u^{m}}{\eta^{m}(\mathbf{x}, \mathbf{u})}
$$

This allows to express the solution $\mathbf{u}=\Theta(\mathbf{x})$ (that may be given in implicit form if some of the infinitesimals $\xi_{i}$ depend on $\mathbf{u}$ ) as

$$
u^{A}=\psi^{A}\left(I_{1}(\mathbf{x}, \mathbf{u}), \ldots, I_{n-1}(\mathbf{x}, \mathbf{u})\right), \quad(A=1,2, \ldots, m)
$$

by substituting (38) into (35) $)_{2}$, a reduced system of differential equations involving $(n-1)$ independent variables (called similarity variables) is obtained. The name similarity variables is due to the fact that the scaling invariance, i.e., the invariance under similarity transformations, was one of the first examples where this procedure has been used systematically.

Note that $I_{1}, I_{2}, \cdots, I_{n-1}, u^{1}, u^{2}, \cdots, u^{m}$ are invariants of the given group, and belong to the set of canonical coordinates. By considering also the variable $I_{n}$, satisfying

$$
\Xi I_{n}=1
$$

we have a complete set of canonical variables for the group characterized by the generator $\Xi$; nevertheless, the determination of $I_{n}$ is not required in this context, because it does not appear explicitly in the reduced system.

If $n=2$, i.e., the system has two independent variables, the reduced system involves only one independent variable, whereupon it is an ordinary differential system.

Example 1 The linear wave equation

$$
\frac{\partial^{2} u}{\partial t^{2}}=\frac{\partial^{2} u}{\partial x_{1}^{2}}+\frac{\partial^{2} u}{\partial x_{2}^{2}}+\frac{\partial^{2} u}{\partial x_{3}^{2}}
$$

admits, among the others, the scaling invariance

$$
\Xi=x_{1} \frac{\partial}{\partial x_{1}}+x_{2} \frac{\partial}{\partial x_{2}}+x_{3} \frac{\partial}{\partial x_{3}}+t \frac{\partial}{\partial t}
$$


The integration of (36) leads to the similarity variables

$$
y_{1}=\frac{x_{1}}{t}, \quad y_{2}=\frac{x_{2}}{t}, \quad y_{3}=\frac{x_{3}}{t}
$$

Taking $u=u\left(y_{1}, y_{2}, y_{3}\right)$, the wave equation becomes a differential equation involving three independent variables:

$$
\frac{\partial^{2} u}{\partial y_{i} \partial y_{k}}\left(\delta_{i k}-y_{i} y_{k}\right)-2 \frac{\partial u}{\partial y_{i}} y_{i}=0
$$

where $\delta_{i k}$ is the Kronecker symbol, and the summation over repeated indices holds.

Since differential equations can admit more than one symmetry, there are different ways to choose a set of similarity variables by starting from different symmetries. It is also possible to achieve a multiple reduction of variables by using multiple-parameter groups of transformations. When this is possible, there are essentially two ways to obtain such a multiple reduction of independent variables: repeating step by step the procedure used in the case of one-parameter Lie groups for each subgroup considered, or performing the reduction all-in-one.

Reducing step by step the number of variables means performing the following:

1. take a generator of a subgroup (say, $\Xi$, written in terms of the variables involved in the system $\Delta=0)$ and build the associated similarity reduction;

2. write the original system of differential equations $\Delta=0$ in terms of the similarity variables and similarity functions, thus obtaining the reduced system $\widehat{\Delta}=0$;

3. if a further reduction is wanted, set $\Delta=\widehat{\Delta}$, and go to step 1 .

This method works only if each considered subgroup is possessed by the system where the similarity reduction is performed; of course, this is true for the first subgroup considered, but for the subsequent steps this is true only if the subgroup (written in terms of the similarity variables) is inherited by the reduced system.

Example 2 As shown before, the wave equation

$$
\frac{\partial^{2} u}{\partial t^{2}}=\frac{\partial^{2} u}{\partial x_{1}^{2}}+\frac{\partial^{2} u}{\partial x_{2}^{2}}+\frac{\partial^{2} u}{\partial x_{3}^{2}}
$$

can be reduced to

$$
\frac{\partial^{2} u}{\partial y_{i} \partial y_{k}}\left(\delta_{i k}-y_{i} y_{k}\right)-2 \frac{\partial u}{\partial y_{i}} y_{i}=0
$$

by using the scaling group. The generator

$$
\Xi_{2}=x_{2} \frac{\partial}{\partial x_{1}}-x_{1} \frac{\partial}{\partial x_{2}}
$$

of the rotation in the $x_{1} x_{2}$ plane is a second symmetry of the wave equation. In terms of the new variables $y_{i}$ it reads

$$
\Xi_{2}=\Xi_{2}\left(y_{1}\right) \frac{\partial}{\partial y_{1}}+\Xi_{2}\left(y_{2}\right) \frac{\partial}{\partial y_{2}}=y_{2} \frac{\partial}{\partial y_{1}}-y_{1} \frac{\partial}{\partial y_{2}}
$$


which is a symmetry of the reduced wave equation. Its similarity variables are $s=y_{3}$ and $v=y_{1}^{2}+y_{2}^{2}=$ $\left(x_{1}^{2}+x_{2}^{2}\right) / t^{2}$, so that solutions $w=w(s, v)$ may exist and are governed by the new reduced equation

$$
4 v(1-v) \frac{\partial^{2} w}{\partial v^{2}}-4 v s \frac{\partial^{2} w}{\partial v \partial s}-\left(1-s^{2}\right) \frac{\partial^{2} w}{\partial s^{2}}+(4-6 v) \frac{\partial w}{\partial v}-2 s \frac{\partial w}{\partial s}=0
$$

The generator of a pseudo-rotation in the plane $x_{3} t$,

$$
\Xi_{3}=t \frac{\partial}{\partial x_{3}}+x_{3} \frac{\partial}{\partial t}
$$

written in terms of variables $v, s$ and $w$, reads

$$
\Xi_{3}=\Xi_{3}(s) \frac{\partial}{\partial s}+\Xi_{3}(v) \frac{\partial}{\partial v}+\Xi_{3}(w) \frac{\partial}{\partial w}=\left(1-s^{2}\right) \frac{\partial}{\partial s}-2 v s \frac{\partial}{\partial v}
$$

Its similarity variable is $\sigma=v /\left(1-s^{2}\right)=\left(x_{1}^{2}+x_{2}^{2}\right) /\left(t^{2}-x_{3}^{2}\right)$ and the similarity solution $w(\sigma)$ satisfies the equation

$$
\sigma \frac{\partial^{2} w}{\partial \sigma^{2}}+\frac{\partial w}{\partial \sigma}=0
$$

Finally, this ordinary differential equation admits the generator $\Xi_{4}=\partial / \partial w$ (but also the symmetry $\sigma \partial / \partial \sigma$ not inherited from the wave equation), so it can be integrated yielding the particular solution

$$
u=w=a_{1}+a_{2} \ln \sigma=a_{1}+a_{2} \ln \frac{x_{1}^{2}+x_{2}^{2}}{t^{2}-x_{3}^{2}}
$$

where $a_{1}$ and $a_{2}$ are arbitrary constants, of the wave equation.

Example 3 The wave equation in spherical coordinates

$$
\frac{1}{r^{2}} \frac{\partial}{\partial r}\left(r^{2} \frac{\partial u}{\partial r}\right)+\frac{1}{r^{2} \sin \theta} \frac{\partial}{\partial \theta}\left(\sin \theta \frac{\partial u}{\partial \theta}\right)+\frac{1}{r^{2} \sin ^{2} \theta} \frac{\partial^{2} u}{\partial \varphi^{2}}-\frac{\partial^{2} u}{\partial t^{2}}=0
$$

admits, among the others, the group of three-dimensional rotations generated by

$$
\Xi_{1}=\frac{\partial}{\partial \varphi}, \quad \Xi_{2}=\sin \varphi \frac{\partial}{\partial \theta}+\cos \varphi \cot \theta \frac{\partial}{\partial \varphi}, \quad \Xi_{3}=\cos \varphi \frac{\partial}{\partial \theta}-\sin \varphi \cot \theta \frac{\partial}{\partial \varphi}
$$

If we start the reduction procedure with $\Xi_{1}$, then $r, \theta, t$ are the similarity variables and the wave equation reduces to

$$
\frac{1}{r^{2}} \frac{\partial}{\partial r}\left(r^{2} \frac{\partial u}{\partial r}\right)+\frac{1}{r^{2} \sin \theta} \frac{\partial}{\partial \theta}\left(\sin \theta \frac{\partial u}{\partial \theta}\right)-\frac{\partial^{2} u}{\partial t^{2}}=0
$$

This equation does not inherit the symmetries $\Xi_{2}$ and $\Xi_{3}$, and for this reason these generators can not be used for a further reduction.

On the other hand, the wave equation has solutions $u=u(r, t)$, independent of both the coordinates $\varphi$ and $\theta$. 


\subsection{H-Invariant Solutions and Factor Systems}

Let $G$ be the Lie group of transformations admitted by a system of differential equations $\mathcal{E}$ defined by $\boldsymbol{\Delta}=\mathbf{0}$, and let $H \subseteq G$ be an $r$-parameter subgroup.

A solution $\mathbf{u}=\Theta(\mathbf{x})$ of the system $\mathcal{E}$ is referred to as a $H$-invariant solution if the manifold $\mathbf{u}=\boldsymbol{\Theta}(\mathbf{x})$ is an invariant manifold with respect to the $r$-parameter group $H$ generated by the operators

$$
\Xi_{\alpha}=\sum_{i=1}^{n} \xi_{\alpha i} \frac{\partial}{\partial x_{i}}+\sum_{A=1}^{m} \eta_{\alpha}^{A} \frac{\partial}{\partial u^{A}} \quad(\alpha=1, \ldots, r)
$$

this $H$-invariant solution $\mathbf{u}=\boldsymbol{\Theta}(\mathbf{x})$ has to satisfy the differential system at hand and the additional differential equations

$$
\sum_{i=1}^{n} \xi_{\alpha i}(\mathbf{x}, \Theta) \frac{\partial \Theta^{A}}{\partial x_{i}}=\eta_{\alpha}^{A}(\mathbf{x}, \Theta)
$$

with $\alpha=1, \ldots, r$ and $A=1, \ldots, m$. The invariant solutions above discussed are particular instances of $H$-invariant solutions when $r=1$.

Hence, any invariant solution is a solution of the overdetermined system of equations $\mathcal{E}$ and (43). In the general case, any overdetermined system of partial differential equations has to be studied for compatibility. For $H$-invariant solutions this problem is simpler than that in the general case. In fact, due to (43), it has to be

$$
\operatorname{rank} \| \xi_{\alpha i}(\mathbf{x}, \boldsymbol{\Theta}(\mathbf{x})), \eta_{\alpha}^{A}\left(\mathbf{x}, \boldsymbol{\Theta}(\mathbf{x})\|=\operatorname{rank}\| \xi_{\alpha i}(\mathbf{x}, \boldsymbol{\Theta}(\mathbf{x})) \|\right.
$$

Definition 3 A regularly assigned manifold $\Psi \subset \mathbb{R}^{m+n}$ defined by

$$
\Psi_{j}(\mathbf{x}, \mathbf{u})=0, \quad(j=1, \ldots, q)
$$

is called a nonsingular manifold with respect to the group $H$ if

$$
\operatorname{rank}\left\|\left.\left(\xi_{\alpha i}(\mathbf{x}, \mathbf{u}), \eta_{\alpha}^{A}(\mathbf{x}, \mathbf{u})\right)\right|_{\Psi}\right\|=\operatorname{rank} \|\left(\left.\xi_{\alpha i}(\mathbf{x}, \mathbf{u})\right|_{\Psi} \|=r_{\star}, \quad \forall(\mathbf{x}, \mathbf{u}) \in \Psi\right.
$$

Definition 4 An invariant solution

$$
\mathbf{u}=\Theta(\mathbf{x})
$$

is called nonsingular if the manifold defined by $\mathbf{u}=\Theta(\mathbf{x})$ is a nonsingular manifold with respect to the group $H$.

Theorem 5 If $r_{\star}<m+n$, where $r_{\star}$ is given by (44), then there exist $m+n-r_{\star}$ functionally independent invariants $I_{j},\left(j=1, \ldots, N-r_{\star}\right)$ of the symmetries in $H$, and any nonsingular regularly assigned invariant manifold is given by the formula

$$
\Phi_{i}\left(I_{1}(\mathbf{x}, \mathbf{u}), I_{2}(\mathbf{x}, \mathbf{u}), \ldots, I_{m+n-r_{\star}}(\mathbf{x}, \mathbf{u})\right)=0 \quad(i=1, \ldots, q)
$$

Thus, an invariant solution $\mathbf{u}=\Theta(\mathbf{x})$ which is nonsingular, according to the theorem 5 , can be represented by means of $m+n-r_{\star}$ functionally independent invariants. This allows the reduction of the initial system of differential equations $\mathcal{E}$ to a reduced system with fewer independent variables. The following theorem holds true. 
Theorem 6 Let a subgroup $H \subseteq G$ satisfy the condition

$$
\operatorname{rank}\left\|\left(\xi_{\alpha i}, \eta_{\alpha}^{A}\right)\right\|=\operatorname{rank}\left\|\xi_{\alpha i}\right\|=r_{\star} \leq n
$$

Then there are the mappings

$$
\begin{array}{ll}
t_{\ell}=t_{\ell}(\mathbf{x}, \mathbf{u}), & \left(\ell=1, \ldots, n-r_{\star}\right) \\
w^{k}=w^{k}(\mathbf{x}, \mathbf{u}), & (k=1, \ldots, m)
\end{array}
$$

and a system of differential equations $\mathcal{E} / H$ for the functions $\mathbf{W}(\mathbf{t}) \in \mathbb{R}^{m}$ with the independent variables $\mathbf{t}=\left(t_{1}, t_{2}, \ldots, t_{n-r_{\star}}\right)$, satisfying the following properties:

- any nonsingular $H$-invariant solution $\mathbf{u}=\boldsymbol{\Theta}(\mathbf{x})$ can be defined from the functional equations

$$
\mathbf{w}(\mathbf{x}, \mathbf{u}(\mathbf{x}))=\mathbf{W}(\mathbf{t}(\mathbf{x}, \boldsymbol{\Theta}(\mathbf{x})))
$$

where $\mathbf{W}(\mathbf{t})$ is a solution of $\mathcal{E} / H$;

- if a solution $\mathbf{W}(\mathbf{t})$ of the system $\mathcal{E} / H$ produces a manifold in the space $\mathbb{R}^{n}(\mathbf{x}) \times \mathbb{R}^{m}(\mathbf{u})$

$$
\mathbf{w}(\mathbf{x}, \mathbf{u})=\mathbf{W}(\mathbf{t}(\mathbf{x}, \mathbf{u}))
$$

which can be written in the form $\mathbf{u}=\boldsymbol{\Theta}(\mathbf{x})$, then $\mathbf{u}=\boldsymbol{\Theta}(\mathbf{x})$ is a H-invariant solution of the system $\mathcal{E}$.

The system of differential equations $\mathcal{E} / H$ is called a factor system or submodel [7,39]; the number $\sigma=n-r_{\star}$ is the number of the independent variables in the factor system $\mathcal{E} / H$, and it is called the rank of the invariant solution, or the rank of the submodel. According to the theorem, the factor system has two main properties: it possesses all $H$-invariant solutions, and the number of independent variables is less than that in the initial system. It is built algorithmically by means of the following steps:

1. Determine $m+n-r_{\star}=m+\sigma$ independent invariants $I_{k}=I_{k}(\mathbf{x}, \mathbf{u})(k=1, \ldots, m+\sigma)$ of the group $H$. The rank of the Jacobian matrix

$$
\frac{\partial\left(I_{1}, \ldots, I_{m+n-r_{\star}}\right)}{\partial\left(u^{1}, \ldots, u^{m}\right)}
$$

has to be equal to $m$. Without loss of generality, one can order the invariants $I_{1}, \ldots, I_{m+n-r_{\star}}$ in such a way for the first $m$ invariants it is

$$
\operatorname{rank}\left(\frac{\partial\left(I_{1}, \ldots, I_{m}\right)}{\partial\left(u^{1}, \ldots, u^{m}\right)}\right)=m
$$

2. Assume the first $m$ invariants $I_{k}(k=1, \ldots, m)$ to depend on the remaining invariants $I_{k}(k=$ $m+1, \ldots, m+\sigma)$, i.e.,

$$
I_{k}(\mathbf{x}, \mathbf{u})=W^{k}\left(I_{m+1}, \ldots, I_{m+\sigma}\right), \quad(k=1, \ldots, m)
$$

Because of the implicit function theorem and of (45), the relations giving the invariants can be solved with respect to all the dependent variables $u^{A}(A=1, \ldots, m)$. 
3. Substitute the representation of the functions $u^{A}$ into the initial system of partial differential equations to obtain the system of equations for the unknown functions $W^{k}(k=1, \ldots, m)$, i.e., the factor system $\mathcal{E} / H$.

Usually, in the applications, the invariants $I_{m+1}, I_{m+2}, \ldots, I_{m+\sigma}$ can be chosen independent of the dependent variables; in this case the representation of the invariant solution is

$$
u^{A}=\Psi^{A}\left(\mathbf{x}, I_{m+1}, I_{m+2}, \ldots, I_{m+\sigma}\right), \quad(A=1,2, \ldots, m)
$$

where

$$
\Psi^{k}\left(\mathbf{x}, I_{m+1}, \ldots, I_{m+\sigma}\right)=\bar{\Psi}^{k}\left(\mathbf{x}, W^{1}\left(I_{m+1}, \ldots, I_{m+\sigma}\right), \ldots, W^{m}\left(I_{m+1}, I_{m+2}, \ldots, I_{m+\sigma}\right)\right)
$$

for $k=1,2, \ldots, m$.

It can be noticed that, if $r_{\star}=n-1$, the factor system is a system of ordinary differential equations, whereas if $r_{\star}=n$ the factor system is algebraic.

Example 4 By using the full rotation group for a multiple reduction of the wave equation in spherical coordinates, the invariants are $u, r$ and $t$ and they lead directly to the reduced equation

$$
\frac{1}{r^{2}} \frac{\partial}{\partial r}\left(r^{2} \frac{\partial u}{\partial r}\right)-\frac{\partial^{2} u}{\partial t^{2}}=0
$$

It is not necessary to use all possible subgroups to determine all the possible group-invariant solutions, because the transformations of a Lie group admitted by a system of differential equations map any solution into another solution (eventually the same) of the system; this enables us to restrict the search of invariant solutions only to the essential ones.

Definition 5 Two solutions $\mathbf{u}_{1}, \mathbf{u}_{2}$ of a system of differential equations are said to be essentially different if there is no transformation $T$ of the admitted group such that $\mathbf{u}_{2}=T\left(\mathbf{u}_{1}\right)$. In the opposite case they are called nonessentially different.

Since nonessentially different invariant solutions are found from similar subgroups, the problem of the classification of $H$-invariant solutions (i.e., of separating solutions into classes of essentially different solutions) is reduced to the classification of subgroups of the group $G$ up to conjugacy. It can be proved that this problem, in turn, is reduced to the corresponding problem of classification of Lie subalgebras, that can be approached more easily from an algorithmic point of view.

Definition $6([7,10,18,35])$ Two Lie subalgebras $L^{\prime}$ and $L^{\prime \prime}$ of a Lie algebra $L$ are similar if there exists an inner automorphism $\phi \in \operatorname{Int}(L)$ such that $\phi\left(L^{\prime}\right)=L^{\prime \prime}$. Since the similarity between Lie subalgebras is a relation of equivalence, all subalgebras of the given Lie algebra L are decomposed into classes of similar algebras. A set of the representatives of each class is called an optimal system of subalgebras.

Therefore, the knowledge of an optimal system of subalgebras of the principal Lie algebra of a system of differential equations provides a method of classifying $H$-invariant solutions. In [40], a Mathematica ${ }^{\circledR}$ package, SymboLie, has been developed in order to determine optimal systems of Lie subalgebras. 


\subsection{New Solutions from A Known Solution}

The consideration that, under the action of a Lie group of transformations admitted by a differential equation, a solution, which is not invariant with respect to the group, is mapped into a family of solutions, suggests a way of generating new solutions from a known solution. This is especially interesting when one can obtain nontrivial solutions from trivial ones.

Let us consider a one-parameter Lie group of transformations

$$
\mathbf{x}^{\star}=\mathbf{X}(\mathbf{x}, \mathbf{u} ; a), \quad \mathbf{u}^{\star}=\mathbf{U}(\mathbf{x}, \mathbf{u} ; a)
$$

admitted by a system of differential equations $\mathcal{E}$, and let

$$
\mathbf{u}=\Theta(\mathbf{x})
$$

be a solution of the given system $\mathcal{E}$, which is not invariant with respect to the group (47).

The transformation (47) maps a point $(\mathbf{x}, \Theta(\mathbf{x}))$ of the solution $\mathbf{u}=\Theta(\mathbf{x})$ into the point $\left(\mathbf{x}^{\star}, \mathbf{u}^{\star}\right)$ characterized by:

$$
\mathbf{x}^{\star}=\mathbf{X}(\mathbf{x}, \boldsymbol{\Theta}(\mathbf{x}) ; a), \quad \mathbf{u}^{\star}=\mathbf{U}(\mathbf{x}, \boldsymbol{\Theta}(\mathbf{x}) ; a)
$$

For a fixed value of the parameter $a$, one can eliminate $\mathrm{x}$ from (49) by substituting the inverse transformation of (49) ${ }_{1}$,

$$
\mathbf{x}=\mathbf{X}\left(\mathbf{x}^{\star}, \mathbf{u}^{\star} ;-a\right)
$$

into $(49)_{2}$ thus obtaining

$$
\mathbf{u}^{\star}=\mathbf{U}\left(\mathbf{X}\left(\mathbf{x}^{\star}, \mathbf{u}^{\star} ;-a\right), \boldsymbol{\Theta}\left(\mathbf{X}\left(\mathbf{x}^{\star}, \mathbf{u}^{\star} ;-a\right)\right) ; a\right)
$$

Finally, by substituting $\left(\mathbf{x}^{\star}, \mathbf{u}^{\star} ;-a\right)$ with $(\mathbf{x}, \mathbf{u} ; a)$ in (51), one may state the following theorem.

Theorem 7 If $\mathbf{u}=\boldsymbol{\Theta}(\mathbf{x})$ is not an invariant solution of a system $\mathcal{E}$ of differential equations, admitting the group (47), then

$$
\mathbf{u}=\mathbf{U}(\mathbf{X}(\mathbf{x}, \mathbf{u} ; a), \boldsymbol{\Theta}(\mathbf{X}(\mathbf{x}, \mathbf{u} ; a)) ;-a)
$$

implicitly defines a one-parameter family of solutions of the given system.

Example 5 The linear heat equation

admits the Lie group with the generator

$$
\frac{\partial u}{\partial t}-\frac{\partial^{2} u}{\partial x^{2}}=0
$$

$$
\Xi=x t \frac{\partial}{\partial x}+t^{2} \frac{\partial}{\partial t}-\left(\frac{x^{2}}{4}+\frac{t}{2}\right) u \frac{\partial}{\partial u}
$$

Integrating Lie's equations, we obtain the related finite transformation

$$
x^{\star}=\frac{x}{1-a t}, \quad t^{\star}=\frac{t}{1-a t}, \quad u^{\star}=u \sqrt{1-a t} \exp \left(-\frac{a x^{2}}{4(1-a t)}\right)
$$

One has the inverse by exchanging $(x, t, u)$ and $\left(x^{\star}, t^{\star}, u^{\star}\right)$, and replacing $a$ by $-a$; applying this inverse transformation to the trivial solution $u=u_{0}$ ( $u_{0}$ constant) of linear heat equation, the nontrivial solution

$$
u=\frac{u_{0}}{\sqrt{1+a t}} \exp \left(-\frac{a x^{2}}{4(1+a t)}\right)
$$

is immediately generated. 


\subsection{Conservation Laws and Noether's Theorem}

In dealing with differential equations, conservation laws have a deep relevance, since they express the conservation of physical quantities such as mass, momentum, angular momentum, energy, electrical charge. They are also important due to their use in investigating integrability, existence, uniqueness and stability of solutions, or in implementing efficient numerical methods of integration [41,42].

In 1918, Emmy Noether [1] presented her celebrated procedure (Noether's theorem) to find local conservation laws for systems of differential equations arising from a variational principle. Noether proved that a point symmetry of the action functional (action integral) provides a local conservation law through an explicit formula that involves the infinitesimals of the point symmetry and the Lagrangian of the action functional.

A local conservation law of a $k$ th-order system of partial differential equations is a divergence expression

$$
\sum_{i=1}^{n} D_{i} \Phi^{i}\left(\mathbf{x}, \mathbf{u}, \mathbf{u}^{(1)}, \mathbf{u}^{(k-1)}\right)=0
$$

holding for all solutions of the differential system, where $\Phi^{i}(i=1, \ldots, n)$ are called the fluxes of the conservation law.

A conservation law (53) is trivial if each of its fluxes vanishes identically on the solutions of the given system of differential equations, or the conservation law vanishes identically as a differential identity. A trivial conservation law contains no information about a given system of differential equations.

The notion of a trivial conservation law allows for the introduction of equivalent conservation laws and linearly dependent conservation laws.

Definition 7 Two conservation laws $\sum_{i=1}^{n} D_{i} \Phi^{i}=0$ and $\sum_{i=1}^{n} D_{i} \Psi^{i}=0$ are equivalent if

$$
\sum_{i=1}^{n} D_{i}\left(\Phi^{i}-\Psi^{i}\right)=0
$$

is a trivial conservation law.

A set of $l$ conservation laws $\left\{\sum_{i=1}^{n} D_{i} \Phi_{k}^{i}=0: k=1, \ldots, l\right\}$ is linearly dependent if there exists a set of constants $\left\{a_{k}: k=1, \ldots, l\right\}$, not all zero, such that the linear combination

$$
\sum_{i=1}^{n} D_{i}\left(a_{k} \Phi_{k}^{i}\right)=0
$$

is a trivial conservation law. In this case, up to equivalence, one of the conservation laws in the set can be expressed as a linear combination of the remaining ones.

In practice, one is interested in finding linearly independent sets of conservation laws of a given system of differential equations.

In evolutionary problems, when one of the independent variable (say, $x_{n}$ ) is the time $t$, the conservation law has the form

$$
D_{t} \Psi\left(\mathbf{x}, \mathbf{u}, \mathbf{u}^{(1)}, \ldots, \mathbf{u}^{(k-1)}\right)+\sum_{i=1}^{n-1} D_{i} \Phi^{i}\left(\mathbf{x}, \mathbf{u}, \mathbf{u}^{(1)} \mathbf{u}^{(k-1)}\right)=0
$$


where $\Psi$ is referred to as the density, and $\Phi^{i}$ as spatial fluxes of the conservation law. If we are dealing with a system of ordinary differential equations then the conservation law provides a constant of motion.

Consider a functional $\mathcal{J}[\mathbf{x}, \mathbf{u}]$, involving independent variables $\mathbf{x} \in \mathbb{R}^{n}$, dependent variables $\mathbf{u} \in \mathbb{R}^{m}$ and their partial derivatives up to order $k$, defined on a domain $\Omega$,

$$
\mathcal{J}[\mathbf{x}, \mathbf{u}]=\int_{\Omega} \mathcal{L}[\mathbf{x}, \mathbf{u}] d \mathbf{x}=\int_{\Omega} \mathcal{L}\left(\mathbf{x}, \mathbf{u}, \mathbf{u}^{(1)}, \ldots, \mathbf{u}^{(k)}\right) d \mathbf{x}
$$

where $\mathcal{J}[\mathbf{x}, \mathbf{u}]$ is the action integral of the Lagrangian $\mathcal{L}[\mathbf{x}, \mathbf{u}]$. Take an infinitesimal variation of $\mathbf{u}$ given by $\mathbf{u}(\mathbf{x}) \rightarrow \mathbf{u}(\mathbf{x})+a \mathbf{v}(\mathbf{x})$, where $a$ is a small parameter, and $\mathbf{v}(\mathbf{x})$ is any function such that $\mathbf{v}(\mathbf{x})$ and its derivatives up to order $(k-1)$ vanish on the boundary $\partial \Omega$ of the domain $\Omega$. The corresponding variation in the Lagrangian $\mathcal{L}[\mathbf{x}, \mathbf{u}]$ is given by

$$
\begin{aligned}
\delta \mathcal{L} & =\mathcal{L}\left(\mathbf{x}, \mathbf{u}+a \mathbf{v}, \mathbf{u}^{(1)}+a \mathbf{v}^{(1)}, \ldots, \mathbf{u}^{(k)}+a \mathbf{v}^{(k)}\right) \\
& -\mathcal{L}\left(\mathbf{x}, \mathbf{u}, \mathbf{u}^{(1)}, \ldots, \mathbf{u}^{(k)}\right)= \\
& =a\left(\frac{\partial \mathcal{L}[\mathbf{x}, \mathbf{u}]}{\partial u^{A}} v^{A}+\frac{\partial \mathcal{L}[\mathbf{x}, \mathbf{u}]}{\partial u_{j}^{A}} v_{j}^{A}+\ldots+\frac{\partial \mathcal{L}[\mathbf{x}, \mathbf{u}]}{\partial u_{j_{1} \ldots j_{k}}^{A}} v_{j_{1}, \ldots j_{k}}^{A}\right)+O\left(a^{2}\right)
\end{aligned}
$$

where the convention of summation over repeated indices is used.

Repeated integrations by parts yield

$$
\delta \mathcal{L}=a\left(v^{A} E_{u^{A}}(\mathcal{L}[\mathbf{x}, \mathbf{u}])+D_{i} W^{i}[\mathbf{x}, \mathbf{u}, \mathbf{v}]\right)+O\left(a^{2}\right)
$$

where

$$
E_{u^{A}}(\cdot)=\frac{\partial(\cdot)}{\partial u^{A}}-D_{j_{1}}\left(\frac{\partial(\cdot)}{\partial u_{j_{1}}^{A}}\right)+D_{j_{1} j_{2}}\left(\frac{\partial(\cdot)}{\partial u_{j_{1} j_{2}}^{A}}\right)-\ldots+(-1)^{k} D_{j_{1} \ldots j_{k}}\left(\frac{\partial(\cdot)}{\partial u_{j_{1} \ldots j_{k}}^{A}}\right)
$$

is the Euler operator with respect to $u^{A}$, and

$$
\begin{aligned}
W^{i}[\mathbf{x}, \mathbf{u}, \mathbf{v}] & =v^{A}\left(\frac{\partial \mathcal{L}[\mathbf{x}, \mathbf{u}]}{\partial u_{i}^{A}}+\ldots+(-1)^{k-1} D_{j_{1} \ldots j_{k-1}} \frac{\partial \mathcal{L}[\mathbf{x}, \mathbf{u}]}{\partial u_{i j_{1} \ldots j_{k-1}}^{A}}\right) \\
& +v_{j_{1}}^{A}\left(\frac{\partial \mathcal{L}[\mathbf{x}, \mathbf{u}]}{\partial u_{i j_{1}}^{A}}+\ldots+(-1)^{k-2} D_{j_{2} \ldots j_{k-1}} \frac{\partial \mathcal{L}[\mathbf{x}, \mathbf{u}]}{\partial u_{i j_{1} \ldots j_{k-1}}^{A}}\right) \\
& +\ldots+v_{j_{1} \ldots j_{k-1}}^{A} \frac{\partial \mathcal{L}[\mathbf{x}, \mathbf{u}]}{\partial u_{i j_{1} \ldots j_{k-1}}^{A}} .
\end{aligned}
$$

Using the divergence theorem, the variation of the action integral writes

$$
\begin{aligned}
\delta \mathcal{J} & =\mathcal{J}[\mathbf{x}, \mathbf{u}+a \mathbf{v}]-\mathcal{J}[\mathbf{x}, \mathbf{u}]=\int_{\Omega} \delta \mathcal{L} d \mathbf{x}= \\
& =a \int_{\Omega}\left(v^{A} E_{u^{A}}(\mathcal{L}[\mathbf{x}, \mathbf{u}])+D_{i} W^{i}[\mathbf{x}, \mathbf{u}, \mathbf{v}]\right) d \mathbf{x}+O\left(a^{2}\right)= \\
& =a\left(\int_{\Omega} v^{A} E_{u^{A}}(\mathcal{L}[\mathbf{x}, \mathbf{u}]) d \mathbf{x}+\int_{\partial \Omega} W^{i}[\mathbf{x}, \mathbf{u}, \mathbf{v}] \nu_{i} d S\right)+O\left(a^{2}\right)
\end{aligned}
$$

where $\int_{\partial \Omega}$ is the surface integral over the boundary $\partial \Omega$ of the domain $\Omega, \boldsymbol{\nu}=\left(\nu_{1}, \ldots, \nu_{n}\right)$ being the unit outward normal vector to $\partial \Omega$. 
Since $\mathbf{v}(\mathbf{x})$ and its derivatives up to order $(k-1)$ vanish on the boundary $\partial \Omega$, the extremum $\mathbf{u}(\mathbf{x})$ of the action integral $\mathcal{J}[\mathbf{x}, \mathbf{u}]$ has to satisfy the Euler-Lagrange equations

$$
\frac{\partial \mathcal{L}[\mathbf{x}, \mathbf{u}]}{\partial u^{A}}-D_{j_{1}}\left(\frac{\partial \mathcal{L}[\mathbf{x}, \mathbf{u}]}{\partial u_{j_{1}}^{A}}\right)+D_{j_{1} j_{2}}\left(\frac{\partial \mathcal{L}[\mathbf{x}, \mathbf{u}]}{\partial u_{j_{1} j_{2}}^{A}}\right)-\ldots+(-1)^{k} D_{j_{1} \ldots j_{k}}\left(\frac{\partial \mathcal{L}[\mathbf{x}, \mathbf{u}]}{\partial u_{j_{1} \ldots j_{k}}^{A}}\right)=0
$$

$(A=1, \ldots, m)$

To prove Noether's theorem let us consider a Lie group of infinitesimal point transformations

$$
x_{i}^{\star}=x_{i}+a \xi_{i}(\mathbf{x}, \mathbf{u}), \quad u^{A \star}=u^{A}+a \eta^{A}(\mathbf{x}, \mathbf{u})
$$

$(i=1, \ldots, n, A=1, \ldots, m)$, with infinitesimal generator

$$
\Xi=\xi_{i} \frac{\partial}{\partial x_{i}}+\eta^{A} \frac{\partial}{\partial u^{A}}
$$

leaving the action integral invariant, i.e.,

$$
\int_{\Omega^{\star}} \mathcal{L}\left[\mathbf{x}^{\star}, \mathbf{u}^{\star}\right] d \mathbf{x}^{\star}=\int_{\Omega} \mathcal{L}[\mathbf{x}, \mathbf{u}] d \mathbf{x}
$$

where $\Omega^{\star}$ is the image of $\Omega$ under the point transformation. The Jacobian $J$ of the transformation is

$$
J=\operatorname{det}\left\|D_{i} x_{j}^{\star}\right\|=1+a D_{i} \xi_{i}(\mathbf{x}, \mathbf{u})+O\left(a^{2}\right)
$$

whereupon we have

$$
d \mathbf{x}^{\star}=J d \mathbf{x}
$$

Since (62) is a Lie group of point transformations, by using the exponential map of the $k$ th-order infinitesimal generator, it is

$$
\mathcal{L}\left[\mathbf{x}^{\star}, \mathbf{u}^{\star}\right]=\exp \left(a \Xi^{(k)}\right) \mathcal{L}[\mathbf{x}, \mathbf{u}]
$$

Therefore, (62) is a Lie point symmetry of $\mathcal{J}[\mathbf{x}, \mathbf{u}]$ if and only if

$$
\begin{aligned}
0 & \equiv \int_{\Omega}\left(J \exp \left(a \Xi^{(k)}\right)-1\right) \mathcal{L}[\mathbf{x}, \mathbf{u}] d \mathbf{x}= \\
& =a \int_{\Omega}\left(\mathcal{L}[\mathbf{x}, \mathbf{u}]\left(D_{i} \xi_{i}(\mathbf{x}, \mathbf{u})\right)+\Xi^{(k)} \mathcal{L}[\mathbf{x}, \mathbf{u}]\right) d \mathbf{x}+O\left(a^{2}\right)
\end{aligned}
$$

and this implies that the $O(a)$ terms have to vanish, i.e.,

$$
\mathcal{L}[\mathbf{x}, \mathbf{u}]\left(D_{i} \xi_{i}(\mathbf{x}, \mathbf{u})\right)+\Xi^{(k)} \mathcal{L}[\mathbf{x}, \mathbf{u}]=0
$$

A Lie symmetry of $\mathcal{J}[\mathbf{x}, \mathbf{u}]$ is called a variational symmetry. It is worth to be underlined that a variational symmetry (symmetry of action integral) provides a symmetry of the corresponding Euler-Lagrange equations, but the converse is not in general true.

But, the one-parameter Lie group (62) can be equivalently be written in the evolutionary form [19],

$$
x_{i}^{\star}=x_{i}, \quad u^{A \star}=u^{A}+a \widetilde{\eta}^{A}[\mathbf{u}]=u^{A}+a\left(\eta^{A}-\xi_{i} u_{i}^{A}\right)
$$

with infinitesimal generator

$$
\widetilde{\Xi}=\left(\eta^{A}-\xi_{i} u_{i}^{A}\right) \frac{\partial}{\partial u^{A}}
$$


and obvious prolongations.

Using (70) we have

$$
\delta \mathcal{L}[\mathbf{x}, \mathbf{u}]=a \widetilde{\Xi}^{(k)} \mathcal{L}[\mathbf{x}, \mathbf{u}]+O\left(a^{2}\right)
$$

and

$$
\int_{\Omega} \delta \mathcal{L}[\mathbf{x}, \mathbf{u}] d \mathbf{x}=a \int_{\Omega} \widetilde{\Xi}^{(k)} \mathcal{L}[\mathbf{x}, \mathbf{u}] d \mathbf{x}+O\left(a^{2}\right)
$$

the comparison between the latter relation and (60), with $v^{A}(\mathbf{x})=\eta^{A}-\xi_{i} u_{i}^{A}$, provides

$$
\widetilde{\Xi}^{(k)} \mathcal{L}[\mathbf{x}, \mathbf{u}]=\widetilde{\eta}^{A} E_{u^{A}}(\mathcal{L}[\mathbf{x}, \mathbf{u}])+D_{i} W^{i}[\mathbf{x}, \mathbf{u}, \widetilde{\boldsymbol{\eta}}[\mathbf{u}]]
$$

where $W^{i}[\mathbf{x}, \mathbf{u}, \widetilde{\boldsymbol{\eta}}[\mathbf{u}]]$ is obtained, mutatis mutandis, from (59).

The following lemma has a straightforward proof.

Lemma 1 Let $\Xi^{(k)}$ be the kth prolongation of the infinitesimal generator (63) and let $\widetilde{\Xi}^{(k)}$ be the kth prolongation of (71); for an arbitrary function $F[\mathbf{x}, \mathbf{u}]=F\left(\mathbf{x}, \mathbf{u}, \mathbf{u}^{(1)}, \ldots, \mathbf{u}^{(k)}\right)$ the following identity holds:

$$
\Xi^{(k)} F[\mathbf{x}, \mathbf{u}]+F[\mathbf{x}, \mathbf{u}] D_{i} \xi_{i}(\mathbf{x}, \mathbf{u})=\widetilde{\Xi}^{(k)} F[\mathbf{x}, \mathbf{u}]+D_{i}\left(F[\mathbf{x}, \mathbf{u}] \xi_{i}(\mathbf{x}, \mathbf{u})\right)
$$

Now we have all the ingredients to prove Noether's theorem.

Theorem 8 (Noether's theorem) Let us consider a system of differential equations

$$
\Delta\left(\mathbf{x}, \mathbf{u}, \mathbf{u}^{(1)}, \ldots, \mathbf{u}^{(k)}\right)=\mathbf{0}
$$

arising from a variational principle, i.e., a system of the Euler-Lagrange equations whose solutions are extrema of an action integral $\mathcal{J}[\mathbf{x}, \mathbf{u}]$ with Lagrangian $\mathcal{L}[\mathbf{x}, \mathbf{u}]$. If (62) is a Lie point symmetry of $\mathcal{J}[\mathbf{x}, \mathbf{u}]$, and $W_{i}[\mathbf{x}, \mathbf{u}, \mathbf{v}]$ are defined by (59), then

1. the identity

$$
\widetilde{\eta}^{A}[\mathbf{u}] E_{u^{A}}(\mathcal{L}[\mathbf{x}, \mathbf{u}])=-D_{i}\left(\xi_{i}(\mathbf{x u}) \mathcal{L}[\mathbf{x}, \mathbf{u}]+W^{i}[\mathbf{x}, \mathbf{u}, \widetilde{\eta}[\mathbf{u}]]\right)
$$

holds for arbitrary functions $\mathbf{u}(\mathbf{x})$;

2. the local conservation law

$$
D_{i}\left(\xi_{i}(\mathbf{x}, \mathbf{u}) \mathcal{L}[\mathbf{u}]+W^{i}[\mathbf{x}, \mathbf{u}, \widetilde{\eta}[\mathbf{u}]]\right)=0
$$

holds for any solution $\mathbf{u}(\mathbf{x})$ of Euler-Lagrange equations.

Substituting $F[\mathbf{x}, \mathbf{u}]$ with $\mathcal{L}[\mathbf{x}, \mathbf{u}]$ in (75), and using (69), it follows

$$
\widetilde{\Xi}^{(k)} \mathcal{L}[\mathbf{x}, \mathbf{u}]+D_{i}\left(\mathcal{L}[\mathbf{x}, \mathbf{u}] \xi_{i}(\mathbf{x}, \mathbf{u})\right)=0
$$

for arbitrary functions $\mathbf{u}(\mathbf{x})$. Using (74), and assuming $\mathbf{u}(\mathbf{x})$ to be a solution of the Euler-Lagrange equations, the left-hand side of (77) vanishes, whereupon the conservation law (78) arises.

Boyer [43] extended Noether's theorem in order to construct conservation laws arising from invariance under generalized symmetries [10], i.e., symmetries with infinitesimals depending on higher-order derivatives (see [19]). 
Example 6 (Klein-Gordon equation (see [19])) The class of Klein-Gordon wave equations,

$$
u_{t x}+g(u)=0
$$

with a general nonlinear interaction term $g(u)$, can be derived from a variational principle given by the action functional with Lagrangian

$$
\mathcal{L}=-\frac{1}{2} u_{x} u_{t}+h(u), \quad h^{\prime}(u)=g(u)
$$

For an arbitrary $g(u)$, equation (80) is invariant with respect to time and space translation and a scaling group of $x$ and $t$. The generators of these symmetries, written in evolutionary form, are

$$
\widetilde{\Xi}_{1}=u_{t} \frac{\partial}{\partial u}, \quad \widetilde{\Xi}_{2}=u_{x} \frac{\partial}{\partial u}, \quad \widetilde{\Xi}_{3}=\left(t u_{t}-x u_{x}\right) \frac{\partial}{\partial u}
$$

and it is straightforward to verify that they are variational symmetries of the action functional. Applying Noether's theorem, the following conservation laws are easily derived:

$$
\begin{aligned}
& \left(\frac{1}{2} u_{x}^{2}\right)_{t}+(h(u))_{x}=0 \\
& \frac{\partial}{\partial t}(h(u))+\frac{\partial}{\partial x}\left(\frac{1}{2} u_{t}^{2}\right)=0 \\
& \frac{\partial}{\partial t}\left(\frac{1}{2} x u_{x}^{2}-t h(u)\right)+\frac{\partial}{\partial t}\left(-\frac{1}{2} t u_{t}^{2}+x h(u)\right)=0
\end{aligned}
$$

The most important limitation of Noether's theorem for the determination of local conservation laws relies on the fact that it applies to variational systems. For general systems of differential equations, a divergence expression providing a conservation law may be found through a direct approach by taking a linear combinations of the given differential equations with multipliers that are functions of the independent and dependent variables, as well as derivatives (up to some finite order) of the dependent variables $[10,19,44,45]$. In the direct approach the dependent variables (and their derivatives) involved in the system of differential equations, or appearing in the multipliers, are replaced by arbitrary functions (and their derivatives). By their construction, such divergence expressions vanish on all solutions of the given system.

A set of multipliers of order $l,\left\{\Lambda_{\nu}[\mathbf{x}, \mathbf{u}] \equiv \Lambda_{\nu}\left(\mathbf{x}, \mathbf{u}, \mathbf{u}^{(1)}, \ldots, \mathbf{u}^{(l)}\right): \nu=1, \ldots, q\right\}$, provides a divergence expression for the system of differential equations

$$
\Delta_{\nu}[\mathbf{x}, \mathbf{u}] \equiv \Delta_{\nu}\left(\mathbf{x}, \mathbf{u}, \mathbf{u}^{(1)}, \ldots, \mathbf{u}^{(k)}\right)=0, \quad \nu=1, \ldots, q
$$

if the identity

$$
\sum_{\nu=1}^{q} \Lambda_{\nu}[\mathbf{x}, \mathbf{U}] \Delta_{\nu}[\mathbf{x}, \mathbf{U}]=\sum_{i=1}^{n} D_{i} \Phi^{i}[\mathbf{x}, \mathbf{U}]
$$

holds for arbitrary functions $\mathbf{U}(\mathbf{x})$. Then, on the solutions $\mathbf{U}(\mathbf{x})=\mathbf{u}(\mathbf{x})$ of the system (84), if $\Lambda_{\nu}$ is non-singular, one has a conservation law

$$
\sum_{\nu=1}^{q} \Lambda_{\nu}[\mathbf{x}, \mathbf{u}] \Delta_{\nu}[\mathbf{x}, \mathbf{u}] \equiv \sum_{i=1}^{n} D_{i} \Phi^{i}[\mathbf{x}, \mathbf{u}]=0
$$


A multiplier $\Lambda_{\nu}$ is singular if it is a singular function when evaluated on solutions $\mathbf{U}(\mathbf{x})=\mathbf{u}(\mathbf{x})$ of the system (84).

The direct approach provides useful provided one is able

1. to derive a set of determining equations to find all sets of multipliers of the system (84) yielding its nontrivial conservation laws;

2. to establish the conditions for which all nontrivial conservation laws arise from sets of local multipliers and viceversa;

3. to construct the fluxes of a conservation law arising from a given set of multipliers.

The answer to the first item involves the use of the Euler operators (58). In fact, a direct computation shows that the Euler operators (58) annihilate any divergence expression, and that the only scalar expressions annihilated by Euler operators are divergence expressions. Then, the following theorem arises.

Theorem 9 (See [19]) A set of non-singular multipliers $\left\{\Lambda_{\nu}[\mathbf{x}, \mathbf{U}]: \nu=1, \ldots, q\right\}$ yields a local conservation law for the system $\left\{\Delta_{\nu}[\mathbf{U}]=0: \nu=1, \ldots, q\right\}$ if and only if the set of identities

$$
E_{U^{A}}\left(\Lambda_{\nu}[\mathbf{x}, \mathbf{U}] \Delta_{\nu}[\mathbf{x}, \mathbf{U}]\right)=0, \quad A=1, \ldots, m
$$

holds for arbitrary functions $\mathbf{U}(\mathbf{x})$.

The set of equations (85) yields the set of linear determining equations to find all sets of conservation law multipliers of the given system of differential equations provided that multipliers of all orders $l=1,2, \ldots$ are considered. Since equations (85) hold for arbitrary $\mathbf{U}(\mathbf{x})$, it follows that one can treat each component of $\mathbf{U}$ together to its derivatives as independent variables, whereupon the linear system of partial differential equations (85) splits into an overdetermined linear system of determining equations whose solutions are the sets of multipliers.

To answer to the second item it is necessary to have the system (84) solved with respect to some leading derivatives, as required by Lie's algorithm for the determination of the admitted symmetries. In such a case, all conservation laws, up to equivalence, arise from local multipliers that are the solutions of the determining equations (85) [19].

Finally, the answer to the third item corresponds formally to the problem of inversion of the divergence differential operator. Several ways of finding the fluxes of conservation laws from a known set of multipliers exist. A direct method (see [19]) converts the set of determining equations for multipliers into the set of determining equations to be solved for the fluxes $\Phi^{i}[\mathbf{x}, \mathbf{U}]$.

Remarkably, if the system of differential equations is variational then its conservation law multipliers correspond to variational symmetries.

The interest reader my refer to [19] for more details as well as worked examples.

\section{Transformation to Autonomous Form}

Lie point symmetries of differential equations can be also used to construct a mapping from a given (source) system of differential equations to another (target) suitable system of differential equations. 
Such a mapping (if it exists) needs not be a group transformation; moreover, any infinitesimal generator admitted by the source system of differential equations has to be mapped to an infinitesimal generator admitted by the target system of differential equations [11].

If the mapping is one-to-one (invertible) then the mapping must establish a one-to-one correspondence between infinitesimal generators of the source and target system of differential equations. In other words, the Lie algebra of infinitesimal operators of the target system of differential equations has to be isomorphic to the Lie algebra of infinitesimal operators of the source system of differential equations. On the contrary, if the mapping from the source system to the target one is allowed to be non-invertible, then it is not necessary that there be a one-to-one correspondence between Lie Algebras of infinitesimal operators of source and target systems of differential equations.

But such a non-invertible mapping must take any infinitesimal operator admitted by the source system into an infinitesimal operator (which could be a null operator) admitted by the target one. More precisely, the mapping must establish a homomorphism between any Lie algebra of infinitesimal operators of the source system and a Lie Algebra of infinitesimal operators of the target system.

As a first case, we consider whether a transformation of a nonautonomous system of differential equations to autonomous form, i.e., a form in which the independent variables do not appear explicitly, exists. In [46] a theorem giving the necessary and sufficient conditions for the existence of an invertible transformation mapping a nonautonomous system of partial differential equations involving two independent variables to autonomous form has been given. For a general nonautonomous system of partial differential equations the following theorem holds (see also [47]).

Theorem 10 The system of differential equations of order $k$

$$
\Delta\left(\mathbf{x}, \mathbf{u}, \mathbf{u}^{(1)}, \ldots, \mathbf{u}^{(k)}\right)=0
$$

where $\mathbf{x} \in \mathbb{R}^{n}$ and $\mathbf{u} \in \mathbb{R}^{m}$, can be transformed by an invertible point transformation, say

$$
\mathbf{y}=\mathbf{Y}(\mathbf{x}, \mathbf{u}), \quad \mathbf{w}=\mathbf{W}(\mathbf{x}, \mathbf{u})
$$

to the autonomous equivalent form

$$
\widetilde{\Delta}\left(\mathbf{w}, \mathbf{w}^{(1)}, \ldots, \mathbf{w}^{(k)}\right)=0
$$

if and only if it is left invariant by $n$ Lie groups of point transformations whose infinitesimal operators $\Xi_{i}(i=1, \ldots, n)$ give a distribution of rank $n$, and satisfy the conditions:

$$
\left[\Xi_{i}, \Xi_{j}\right]=0, \quad(i, j=1, \ldots, n)
$$

that is, the operators $\Xi_{i}$ span an $n$-dimensional Abelian Lie algebra.

Suppose that (86) is invariant with respect to the $n$ Lie groups whose operators generate an Abelian Lie algebra. Let us introduce the canonical variables

$$
y_{1}, \quad y_{i_{1}}^{1}\left(i_{1}=2, \ldots, n\right), \quad v_{A}^{1}(A=1, \ldots, m)
$$

such that

$$
\Xi_{1} y_{1}=1, \quad \Xi_{1} y_{i_{1}}^{1}=0, \quad \Xi_{1} v_{A}^{1}=0
$$


as a consequence, $\Xi_{1}$ takes the form

$$
\Xi_{1}=\frac{\partial}{\partial y_{1}}
$$

i.e., it corresponds to a translation in the variable $y_{1}$.

Since $\left[\Xi_{1}, \Xi_{2}\right]=0$ it follows:

$$
\Xi_{1}\left(\Xi_{2} y_{1}\right)=\Xi_{2}\left(\Xi_{1} y_{1}\right)=0, \quad \Xi_{1}\left(\Xi_{2} y_{i_{1}}^{1}\right)=\Xi_{2}\left(\Xi_{1} y_{i_{1}}^{1}\right)=0, \quad \Xi_{1}\left(\Xi_{2} v_{A}^{1}\right)=\Xi_{2}\left(\Xi_{1} v_{A}^{1}\right)=0
$$

Thus $\Xi_{2}$ will depend upon the invariants of $\Xi_{1}$ only and has the form:

$$
\Xi_{2}=\Theta_{1}^{2}\left(y_{j_{1}}^{1}, v_{B}^{1}\right) \frac{\partial}{\partial y_{1}}+\sum_{i_{1}=2}^{n} \Theta_{i_{1}}^{2}\left(y_{j_{1}}^{1}, v_{B}^{1}\right) \frac{\partial}{\partial y_{i_{1}}^{1}}+\sum_{A=1}^{m} \Lambda_{A}^{2}\left(y_{j_{1}}^{1}, v_{B}^{1}\right) \frac{\partial}{\partial v_{A}^{1}}
$$

$\left(j_{1}=2, \ldots, n\right)$.

If $\Theta_{1}^{2} \neq 0$ we need to introduce the variable

$$
y_{1}^{1}=y_{1}+\varphi_{1}\left(y_{i_{1}}^{1}, v_{A}^{1}\right)
$$

where $\varphi_{1}$ satisfies

$$
\Theta_{1}^{2}+\sum_{i_{1}=2}^{n} \Theta_{i_{1}}^{2} \frac{\partial \varphi_{1}}{\partial y_{i_{1}}^{1}}+\sum_{A=1}^{m} \Lambda_{A}^{2} \frac{\partial \varphi_{1}}{\partial v_{A}^{1}}=0
$$

otherwise we take $y_{1}^{1}=y_{1}$.

That enables us to write $\Xi_{1}$ and $\Xi_{2}$ as follows

$$
\Xi_{1}=\frac{\partial}{\partial y_{1}^{1}}, \quad \Xi_{2}=\sum_{i_{1}=2}^{n} \Theta_{i_{1}}^{2}\left(y_{j_{1}}^{1}, v_{B}^{1}\right) \frac{\partial}{\partial y_{i_{1}}^{1}}+\sum_{A=1}^{m} \Lambda_{A}^{2}\left(y_{j_{1}}^{1}, v_{B}^{1}\right) \frac{\partial}{\partial v_{A}^{1}}
$$

where $j_{1}=2, \ldots, n$.

Now let us introduce the canonical variables

$$
y_{2}, \quad y_{i_{2}}^{2}\left(i_{2}=3, \ldots, n\right), \quad v_{A}^{2}(A=1, \ldots, m)
$$

related to the operator $\Xi_{2}$ such that it results

$$
\Xi_{2}=\frac{\partial}{\partial y_{2}}
$$

Since $\Xi_{3}$ commutes with $\Xi_{1}$ and $\Xi_{2}$, it writes under the form:

$$
\Xi_{3}=\Theta_{1}^{3}\left(y_{j_{2}}^{2}, v_{B}^{2}\right) \frac{\partial}{\partial y_{1}^{1}}+\Theta_{2}^{3}\left(y_{j_{2}}^{2}, v_{B}^{2}\right) \frac{\partial}{\partial y_{2}}+\sum_{i_{2}=2}^{n} \Theta_{i_{2}}^{3}\left(y_{j_{2}}^{2}, v_{B}^{2}\right) \frac{\partial}{\partial y_{i_{2}}^{2}}+\sum_{A=1}^{m} \Lambda_{A}^{3}\left(y_{j_{2}}^{2}, v_{B}^{2}\right) \frac{\partial}{\partial v_{A}^{2}}
$$

where $j_{2}=3, \ldots, n$.

If $\Theta_{1}^{3} \neq 0$ we need to introduce the variable

$$
y_{1}^{2}=y_{1}^{1}+\varphi_{1}^{1}\left(y_{i_{2}}^{2}, v_{A}^{2}\right)
$$

with $\varphi_{1}^{1}$ such that

$$
\Theta_{1}^{3}+\sum_{i_{2}=2}^{n} \Theta_{i_{2}}^{3} \frac{\partial \varphi_{1}^{1}}{\partial y_{i_{2}}^{2}}+\sum_{A=1}^{m} \Lambda_{A}^{3} \frac{\partial \varphi_{1}^{1}}{\partial v_{A}^{2}}=0
$$


otherwise we take $y_{1}^{2}=y_{1}^{1}$; moreover, if $\Theta_{2}^{3} \neq 0$ we need to introduce the variable

$$
y_{2}^{2}=y_{2}+\varphi_{2}^{1}\left(y_{i_{2}}^{2}, v_{A}^{2}\right)
$$

with $\varphi_{2}^{1}$ such that

$$
\Theta_{2}^{3}+\sum_{i_{2}=2}^{n} \Theta_{i_{2}}^{3} \frac{\partial \varphi_{2}^{1}}{\partial y_{i_{2}}^{2}}+\sum_{A=1}^{m} \Lambda_{A}^{3} \frac{\partial \varphi_{2}^{1}}{\partial v_{A}^{2}}=0
$$

otherwise we take $y_{2}^{2}=y_{2}$.

Thus we are able to write $\Xi_{1}, \Xi_{2}$ and $\Xi_{3}$ as follows

$$
\Xi_{1}=\frac{\partial}{\partial y_{1}^{2}}, \quad \Xi_{2}=\frac{\partial}{\partial y_{2}^{2}}, \quad \Xi_{3}=\sum_{i_{2}=3}^{n} \Theta_{i_{2}}^{3}\left(y_{j_{2}}^{2}, v_{B}^{2}\right) \frac{\partial}{\partial y_{i_{2}}^{2}}+\sum_{A=1}^{m} \Lambda_{A}^{3}\left(y_{j_{2}}^{2}, v_{B}^{2}\right) \frac{\partial}{\partial v_{A}^{2}}
$$

By iterating the procedure for the remaining operators, at the generic $k$ th step we introduce the canonical variables

$$
y_{k}, \quad y_{i_{k}}^{k}\left(i_{k}=k+1, \ldots, n\right), \quad v_{A}^{k}(A=1, \ldots, m)
$$

such that the operators $\Xi_{1}, \Xi_{2}, \ldots, \Xi_{k}, \Xi_{k+1}$ write:

$$
\begin{aligned}
\Xi_{1} & =\frac{\partial}{\partial y_{1}^{k-1}}, \quad \Xi_{2}=\frac{\partial}{\partial y_{2}^{k-1}}, \quad \ldots \ldots \quad \Xi_{k}=\frac{\partial}{\partial y_{k}} \\
\Xi_{k+1} & =\Theta_{1}^{k+1}\left(y_{j_{k}}^{k}, v_{B}^{k}\right) \frac{\partial}{\partial y_{1}^{k-1}}+\ldots+\Theta_{k}^{k+1}\left(y_{j_{k}}^{k}, v_{B}^{k}\right) \frac{\partial}{\partial y_{k}}+ \\
& +\sum_{i_{k}=k+1}^{n} \Theta_{i_{k}}^{k+1}\left(y_{j_{k}}^{k}, v_{B}^{k}\right) \frac{\partial}{\partial y_{i_{k}}^{k}}+\sum_{A=1}^{m} \Lambda_{A}^{k+1}\left(y_{j_{k}}^{k}, v_{B}^{k}\right) \frac{\partial}{\partial v_{A}^{k}}
\end{aligned}
$$

where $j_{k}=k+1, \ldots, n$.

Finally, the complete application of the described algorithm enables us to write each operator $\Xi_{i}$ in the form

$$
\Xi_{i}=\frac{\partial}{\partial z_{i}}
$$

where we have set $z_{i}=y_{i}^{n}(i=1, \ldots, n-1)$ and $z_{n}=y_{n}$; moreover, we set $w_{A}=v_{A}^{n}$.

By combining all the transformations involved at the different steps of the algorithm we determine a variable transformation like (87) allowing to write the system (86) in the autonomous form (88).

Conversely, if the nonautonomous system (86) can be mapped by the invertible point transformation (87) to the autonomous system (88), then, since the latter admits the $n$ operators $\frac{\partial}{\partial z_{i}}$, spanning an $n$-dimensional Abelian Lie algebra, then it follows that also the system (86) must admit $n$ infinitesimal operators generating an $n$-dimensional Abelian Lie algebra.

This theorems provides useful for instance when one is facing nonlinear propagation of discontinuity waves [48] in states which are not constant, or when special geometrical assumptions (spherical or cylindrical symmetry) are intrinsic to the studied problem. Some earlier applications to relevant physical applications can be found in [49,50]. In [50] and in [51] the point explosion problem in spherical symmetry has been considered for the equations of ideal gas dynamics and of a monatomic gas in the context of the Extended Thermodynamics [52], respectively. Other applications can be found for instance in [53-56]. 


\subsection{Reduction to Autonomous Form: Applications}

To illustrate the use of theorem 10 we give two simple examples.

Example 7 (Cylindrical KdV Equation) Consider the cylindrical Korteweg-deVries equation

$$
\frac{\partial u}{\partial t}+u \frac{\partial u}{\partial x}+\frac{\partial^{3} u}{\partial x^{3}}+\frac{u}{2 t}=0
$$

which is said to describe the "cylindrical solitons"; it is known [57] that it can be reduced to the classical KdV equation. We will present here this reduction as a particular case of the general procedure presented before.

The equation (96) is left invariant, among the others, by the Lie groups of point transformations generated by the following two commuting operators:

$$
\Xi_{1}=t^{1 / 2} \frac{\partial}{\partial x}+\frac{1}{2} t^{-1 / 2} \frac{\partial}{\partial u}, \quad \Xi_{2}=t^{3 / 2} \frac{\partial}{\partial t}+\frac{1}{2} x t^{1 / 2} \frac{\partial}{\partial x}+\left(\frac{1}{4} x t^{-1 / 2}-u t^{1 / 2}\right) \frac{\partial}{\partial u}
$$

The canonical variables with respect to $\Xi_{1}$ are

$$
t, \quad \xi=\frac{x}{t^{1 / 2}}, \quad U=t u-\frac{x}{2}
$$

hence, $\Xi_{1}$ and $\Xi_{2}$ become

$$
\Xi_{1}=\frac{\partial}{\partial \xi}, \quad \Xi_{2}=t^{3 / 2} \frac{\partial}{\partial t}
$$

whereupon, by introducing

$$
\tau=-2 t^{-1 / 2} \quad \Rightarrow \quad \Xi_{2}=\frac{\partial}{\partial \tau}
$$

the equation (96) writes as

$$
\frac{\partial U}{\partial \tau}+U \frac{\partial U}{\partial \xi}+\frac{\partial^{3} U}{\partial \xi^{3}}=0
$$

that is the classical KdV equation. Equation (101) has the well known "soliton" solution

$$
U=12 \operatorname{sech}^{2}(\xi-4 \tau)
$$

to which will correspond the solution

$$
u=\frac{x}{2 t}+\frac{12}{t} \operatorname{sech}^{2}\left(\frac{x+8}{\sqrt{t}}\right)
$$

to the cylindrical $\mathrm{KdV}$ equation.

Example 8 (Axi-symmetric gas dynamics) Consider the equations of axi-symmetric gas dynamics:

$$
\begin{aligned}
& \frac{\partial \rho}{\partial t}+\frac{\partial(\rho u)}{\partial x}+\frac{\nu \rho u}{x}=0 \\
& \rho\left(\frac{\partial u}{\partial t}+u \frac{\partial u}{\partial x}\right)+\frac{\partial p}{\partial x}=0 \\
& \frac{\partial p}{\partial t}+u \frac{\partial p}{\partial x}+\gamma p \frac{\partial u}{\partial x}+\frac{\gamma \nu p u}{x}=0
\end{aligned}
$$


where $\rho(x, t)$ is the mass density, $u(x, t)$ the velocity and $p(x, t)$ the pressure; moreover, $\gamma$ is the adiabatic index, and $\nu$ determines the symmetry ( $\nu=0$ plane, $\nu=1$ cylindrical, and $\nu=2$ spherical symmetry).

It is easy to verify that this system is left invariant by the Lie transformations having the following infinitesimal operators:

$$
\begin{array}{lll}
\Xi_{1}=\rho \frac{\partial}{\partial \rho}+p \frac{\partial}{\partial p}, & \Xi_{2}=t \frac{\partial}{\partial t} \\
\Xi_{3}=x \frac{\partial}{\partial x}+u \frac{\partial}{\partial u}+2 p \frac{\partial}{\partial p}, & \Xi_{4}=\frac{\partial}{\partial t}
\end{array}
$$

We build the infinitesimal operators ( $\alpha, \beta$ constants):

$$
\begin{aligned}
& \Xi_{A}=\Xi_{2}+\alpha \Xi_{1}=t \frac{\partial}{\partial t}+\alpha \rho \frac{\partial}{\partial \rho}-u \frac{\partial}{\partial u}+(\alpha-2) p \frac{\partial}{\partial p} \\
& \Xi_{B}=\Xi_{3}+\beta \Xi_{1}=x \frac{\partial}{\partial x}+\beta \rho \frac{\partial}{\partial \rho}+u \frac{\partial}{\partial u}+(\beta+2) p \frac{\partial}{\partial p}
\end{aligned}
$$

that are commuting.

We are led, through the application of theorem 10, to the transformations

$$
\begin{aligned}
& \eta=\ln x, \quad \tau=\ln t \\
& \rho(x, t)=x^{\beta} t^{\alpha} R(\eta, \tau) \\
& u(x, t)=x t^{-1} U(\eta, \tau) \\
& p(x, t)=x^{\beta+2} t^{\alpha-2} P(\eta, \tau)
\end{aligned}
$$

in terms of which the basic system reduces to the following autonomous form:

$$
\begin{aligned}
& \frac{\partial R}{\partial \tau}+\frac{\partial(R U)}{\partial \eta}+(\beta+1+\nu) R U+\alpha R=0 \\
& R\left(\frac{\partial U}{\partial \tau}+U \frac{\partial U}{\partial \eta}\right)+\frac{\partial P}{\partial \eta}+R U(U-1)+(\beta+2) P=0 \\
& \frac{\partial P}{\partial \tau}+U \frac{\partial P}{\partial \eta}+\gamma P \frac{\partial U}{\partial \eta}+(\beta+2+\gamma(\nu+1)) P U+(\alpha-2) P=0
\end{aligned}
$$

The form of this system allows for the possibility of looking for a constant solution

$$
R=R_{0}, \quad U=U_{0}, \quad P=P_{0}
$$

provided that we satisfy the following system of algebraic equations:

$$
\begin{aligned}
& \alpha+(\beta+1+\nu) U_{0}=0 \\
& R_{0} U_{0}\left(U_{0}-1\right)+(\beta+2) P_{0}=0 \\
& (\alpha-2)+(\beta+2+\gamma(\nu+1)) U_{0}=0
\end{aligned}
$$

To the constant solution there corresponds the following non-constant solution of the original system:

$$
\begin{aligned}
\rho(x, t) & =R_{0} x^{\beta} t^{\alpha} \\
u(x, t) & =\frac{-\alpha}{\beta+\nu+1} \frac{x}{t} \\
p(x, t) & =R_{0} \frac{-\alpha(\alpha+\beta+\nu+1)}{(\beta+\nu+1)^{2}(\beta+2)} x^{\beta+2} t^{\alpha-2}
\end{aligned}
$$


where $R_{0}$ and $\beta$ are arbitrary constants whereas

$$
\alpha=\frac{-2(\beta+\nu+1)}{(\gamma+1)+\nu(\gamma-1)}
$$

Note that the solution still exists when $\nu=0$ (plane symmetry). In such a case the transformations reduce an autonomous system to a different autonomous system possessing non-trivial constant solutions which are non-constant in the original physical variables.

In spherical symmetry this solution generalizes the classical Sedov solution [6] for the point explosion problem, the latter being recovered when

$$
\alpha=-\beta=\frac{2}{1-\gamma} \quad \text { and } \quad \nu=2
$$

Another example where the theorem applies is provided by the Navier-Stokes-Fourier equations for a gas in rotation about a fixed axis with a constant angular velocity. The ordinary thermodynamics of viscous and heat conducting monatomic gases can be obtained starting from the balance equations for the first 13 moments and using a formal iterative scheme analogous to the Maxwellian iteration [52,58]. In this case we have only five fields (the gas density, the three components of velocity and the temperature), and four independent variables. The equations (which are nonautonomous) admit a 12-dimensional Lie algebras of point symmetries, and, using two different 4-dimensional Abelian subalgebras, reductions to autonomous form can be obtained [59].

\section{Transformation to Linear Form}

Lie symmetries spanning an infinite-dimensional Lie algebra provide useful to transform nonlinear partial differential equations to linear form. In this section we restrict ourselves to the case in which:

- both the source and target system of partial differential equations are first order systems;

- the mapping is a one-to-one transformation;

- the target system of partial differential equations is linear.

Necessary and sufficient conditions for the existence of invertible mappings linking a nonlinear system of first order partial differential equations with a linear system of differential equations have been given by Kumei and Bluman [60] (see also [11]). However, the proof they give does not seem to involve "natural" conditions. A more "natural" proof has been given in [61] and it involves the introduction of the canonical variables related to some infinitesimal operators (in general not admitted by the source system of partial differential equations) but whose linear combination (with multipliers given by arbitrary functions that are solution of a linear system of partial differential equations) is an admitted group of the source system.

Theorem 11 ([61]) The nonlinear first order system of partial differential equations

$$
\Delta\left(\mathbf{x}, \mathbf{u}, \mathbf{u}^{(1)}\right)=0
$$


where $\mathbf{x} \in \mathbb{R}^{n}, \mathbf{u} \in \mathbb{R}^{m}$, can be transformed to the linear form

$$
L(\mathbf{z})[\mathbf{w}]=\mathbf{g}(\mathbf{z})
$$

where $L(\mathbf{z})$ is a linear first order differential operator, by means of the invertible point transformation

$$
\mathbf{z}=\mathbf{Z}(\mathbf{x}, \mathbf{u}), \quad \mathbf{w}=\mathbf{W}(\mathbf{x}, \mathbf{u})
$$

if and only if it is left invariant by a Lie group of point transformations whose infinitesimal operator has the form

$$
\Xi=\sum_{A=1}^{m} F_{A}(\mathbf{z}) \Xi_{A}
$$

where

$$
\Xi_{A}=\sum_{\alpha=1}^{n} \xi_{A}^{\alpha}(\mathbf{x}, \mathbf{u}) \frac{\partial}{\partial x_{\alpha}}+\sum_{C=1}^{m} \eta_{A}^{C}(\mathbf{x}, \mathbf{u}) \frac{\partial}{\partial u_{C}}
$$

with $\xi_{A}^{\alpha}(\mathbf{x}, \mathbf{u}), \eta_{A}^{C}(\mathbf{x}, \mathbf{u})$ being specific functions of their arguments, and the functions $F_{A}(\mathbf{z})$ satisfying the linear system

$$
L(\mathbf{z})\left[F_{A}\right]=0
$$

along with the conditions

$$
\Xi_{A} \mathbf{z}=\mathbf{0}, \quad\left[\Xi_{A}, \Xi_{B}\right]=0, \quad A, B=1, \ldots, m
$$

First we introduce the canonical variables with respect to $\Xi_{1}$. A possible choice is represented by

$$
\mathbf{z}, \quad u_{A_{1}}^{1}\left(A_{1}=2, \ldots, N\right), \quad v_{1}
$$

such that:

$$
\Xi_{1} \mathbf{z}=\mathbf{0}, \quad \Xi_{1} u_{A_{1}}^{1}=0, \quad \Xi_{1} v_{1}=1
$$

whereupon $\Xi_{1}$ becomes

$$
\Xi_{1}=\frac{\partial}{\partial v_{1}}
$$

Since $\left[\Xi_{1}, \Xi_{2}\right]=0$, it is

$$
\Xi_{1}\left(\Xi_{2} u_{A_{1}}^{1}\right)=\Xi_{2}\left(\Xi_{1} u_{A_{1}}^{1}\right)=0, \quad \Xi_{1}\left(\Xi_{2} v_{1}\right)=\Xi_{2}\left(\Xi_{1} v_{1}\right)=0, \quad \Xi_{2}\left(z_{\alpha}\right)=0
$$

moreover, since $\mathbf{z}$ are invariant with respect to $\Xi_{2}$, the latter operator in the new variables has the form

$$
\Xi_{2}=\Theta_{1}^{2}\left(\mathbf{z}, u_{B_{1}}^{1}\right), \frac{\partial}{\partial v_{1}}+\sum_{A_{1}=2}^{m} \Lambda_{A_{1}}^{2}\left(\mathbf{z}, u_{B_{1}}^{1}\right) \frac{\partial}{\partial u_{A_{1}}^{1}}
$$

$\left(B_{1}=2, \ldots, m\right)$.

If $\Theta_{1}^{2} \neq 0$ we need to introduce the variable

$$
v_{1}^{1}=v_{1}+\varphi_{1}\left(\mathbf{z}, u_{A_{1}}^{1}\right)
$$


with $\varphi_{1}$ such that

$$
\Theta_{1}^{2}+\sum_{A_{1}=2}^{m} \Lambda_{A_{1}}^{2} \frac{\partial \varphi_{1}}{\partial u_{A_{1}}^{1}}=0
$$

otherwise we take $v_{1}^{1}=v_{1}$.

Then we introduce the canonical variables with respect to $\Xi_{2}$; a possible choice is given by

$$
\mathbf{z}, v_{1}^{1}, u_{A_{2}}^{2}
$$

$\left(A_{2}=3, \ldots, m\right)$ and $v_{2}$ such that:

$$
\Xi_{2} u_{A_{2}}^{2}=0, \quad \Xi_{2} v_{2}=1
$$

Thus we have:

$$
\Xi_{1}=\frac{\partial}{\partial v_{1}^{1}}, \quad \Xi_{2}=\frac{\partial}{\partial v_{2}}
$$

By iterating the procedure for the remaining operators, at the generic $k$ th step we have introduced the canonical variables

$$
\mathbf{z}, \quad v_{1}^{k-1}, \ldots, v_{k-1}^{k-1}, v_{A_{k}}^{k}
$$

$\left(A_{k}=k+1, \ldots, m\right)$ and $v^{k}$ such that the operators $\Xi_{1}, \Xi_{2}, \ldots, \Xi_{k}, \Xi_{k+1}$ write:

$$
\begin{aligned}
\Xi_{1} & =\frac{\partial}{\partial v_{1}^{k-1}}, \quad \Xi_{2}=\frac{\partial}{\partial v_{2}^{k-1}}, \quad \ldots \quad \Xi_{k}=\frac{\partial}{\partial v_{k}} \\
\Xi_{k+1} & =\Theta_{1}^{k+1}\left(\mathbf{z}, u_{B_{k}}^{k}\right) \frac{\partial}{\partial v_{1}^{k-1}}+\ldots+\Theta_{k}^{k+1}\left(\mathbf{z}, u_{B_{k}}^{k}\right) \frac{\partial}{\partial v_{k}}+\sum_{A_{k}=k+1}^{n} \Lambda_{A_{k}}^{k+1}\left(\mathbf{z}, u_{B_{k}}^{k}\right) \frac{\partial}{\partial v_{A_{k}}^{k}}
\end{aligned}
$$

Finally, after the complete application of the described algorithm, each operator $\Xi_{A}$ becomes

$$
\Xi_{A}=\frac{\partial}{\partial w_{A}}
$$

where we have set $w_{A}=v_{A}^{m}(A=1, \ldots, m-1)$ and $w_{m}=v_{m}$.

Consequently, the operator $\Xi$ (admitted by the source system of partial differential equations) becomes

$$
\Xi=\sum_{A=1}^{m} F_{A}(\mathbf{z}) \frac{\partial}{\partial w_{A}}
$$

which corresponds to the linear superposition of solutions.

Combining all the transformations involved at the different steps of the algorithm, the transformation

$$
\mathbf{z}=\mathbf{Z}(\mathbf{x}, \mathbf{u}), \quad \mathbf{w}=\mathbf{W}(\mathbf{x}, \mathbf{u})
$$

allows us to write the source system of partial differential equations in the form

$$
\widetilde{\Delta}\left(\mathbf{z}, \mathbf{w}, \mathbf{w}^{(1)}\right)=0
$$

that must be linear.

Conversely, the necessary condition is provided by a theorem of Müller and Matschat [62] proving that for systems of first order partial differential equations a linearizing transformation, if it exists, is a point transformation. 


\subsection{Transformation to Linear Form: Applications}

Some examples where the above theorem applies are given; other examples may be found in [61].

Example 9 (Hodograph transformation) [11,60] The $2 \times 2$ quasilinear homogeneous system of partial differential equations

$$
A_{1}(\mathbf{u}) \frac{\partial \mathbf{u}}{\partial x_{1}} A_{2}(\mathbf{u}) \frac{\partial \mathbf{u}}{\partial x_{2}}=\mathbf{0}, \quad \mathbf{u}=\left[u_{1}, u_{2}\right]^{T}
$$

where $A_{1}$ and $A_{2}$ are $2 \times 2$ matrices with entries arbitrary functions of $\mathbf{u}$, admits the infinitesimal operator

$$
\Xi=F_{1}\left(u_{1}, u_{2}\right) \frac{\partial}{\partial x_{1}}+F_{2}\left(u_{1}, u_{2}\right) \frac{\partial}{\partial x_{2}}
$$

where $F_{1}$ and $F_{2}$ satisfy the linear system

$$
A_{2}(\mathbf{u}) J \frac{\partial \mathbf{F}}{\partial u_{1}}-A_{1}(\mathbf{u}) J \frac{\partial \mathbf{F}}{\partial u_{2}}=\mathbf{0}, \quad \mathbf{F}=\left[F_{1}, F_{2}\right]^{T}, \quad J=\left[\begin{array}{ll}
0 & 1 \\
-1 & 0
\end{array}\right]
$$

Through the transformation

$$
z_{1}=u_{1}, \quad z_{2}=u_{2}, \quad w_{1}=x_{1}, \quad w_{2}=x_{2}
$$

the source system is mapped to the linear system

$$
A_{2}(\mathbf{z}) J \frac{\partial \mathbf{w}}{\partial z_{1}}-A_{1}(\mathbf{z}) J \frac{\partial \mathbf{w}}{\partial z_{2}}=\mathbf{0}, \quad \mathbf{w}=\left[w_{1}, w_{2}\right]^{T}
$$

The transformation here considered is the well known hodograph transformation that is one-to-one if $\operatorname{det}\left\|\frac{\partial\left(u_{1}, u_{2}\right)}{\partial\left(x_{1}, x_{2}\right)}\right\| \neq 0$.

Example 10 (Nonlinear diffusion equation) [63] The equation

$$
\frac{\partial u}{\partial x_{1}}-\frac{\partial}{\partial x_{2}}\left(\frac{1}{u^{2}} \frac{\partial u}{\partial x_{2}}\right)-\frac{\alpha}{u^{2}} \frac{\partial u}{\partial x_{2}}=0
$$

occurring in two-phase flows in porous media, can be written as a first order system:

$$
\frac{\partial u_{2}}{\partial x_{2}}=u_{1}, \quad \frac{\partial u_{2}}{\partial x_{1}}=\frac{1}{\left(u_{1}\right)^{2}} \frac{\partial u_{1}}{\partial x_{2}}+\frac{\alpha}{u_{1}}
$$

where $u_{1}=u$. This system admits the operator

$$
\Xi=F_{1}\left(x_{1}, u_{2}\right) \Xi_{1}+F_{2}\left(x_{1}, u_{2}\right) \Xi_{2}
$$

where

$$
\Xi_{1}=\exp \left(-\alpha x_{2}\right)\left(\frac{1}{\alpha} \frac{\partial}{\partial x_{2}}+u_{1} \frac{\partial}{\partial u_{1}}\right), \quad \Xi_{2}=-\frac{\left(u_{1}\right)^{2} \exp \left(-\alpha x_{2}\right)}{\alpha} \frac{\partial}{\partial u_{1}}
$$

with $F_{1}$ and $F_{2}$ solutions of the linear system

$$
\frac{\partial F_{1}}{\partial u_{2}}=F_{2}, \quad \frac{\partial F_{2}}{\partial u_{2}}=\frac{\partial F_{1}}{\partial x_{1}}
$$


Introducing the canonical variables with respect to $\Xi_{1}$

$$
\begin{aligned}
& z_{1}=x_{1}, \quad z_{2}=u_{2}, \\
& w_{1}=\exp \left(\alpha x_{2}\right), \quad v_{2}=u_{1} \exp \left(-\alpha x_{2}\right)
\end{aligned}
$$

we get

$$
\Xi_{1}=\frac{\partial}{\partial w_{1}}, \quad \Xi_{2}=-\frac{\left(v_{2}\right)^{2}}{\alpha} \frac{\partial}{\partial v_{2}}
$$

After the introduction of $w_{2}=\frac{\alpha}{v_{2}}$, we obtain

$$
\Xi=F_{1}\left(z_{1}, z_{2}\right) \frac{\partial}{\partial w_{1}}+F_{2}\left(z_{1}, z_{2}\right) \frac{\partial}{\partial w_{2}}
$$

and the source system reduces to the linear form

$$
\frac{\partial w_{1}}{\partial z_{2}}=w_{2}, \quad \frac{\partial w_{2}}{\partial z_{2}}=\frac{\partial w_{1}}{\partial z_{1}}
$$

that corresponds to the linear heat equation

$$
\frac{\partial w_{1}}{\partial z_{1}}-\frac{\partial^{2} w_{1}}{\partial z_{2}^{2}}=0
$$

Example 11 (Monge-Ampère equation [64]) The Monge-Ampère equation

$$
\kappa_{1}\left(u_{t t} u_{x x}-u_{t x}^{2}\right)+\kappa_{2} u_{t t}+\kappa_{3} u_{t x}+\kappa_{4} u_{x x}+\kappa_{5}=0
$$

where $\kappa_{i}(i=1, \ldots, 5)$ are constant, through the positions

$$
t=x_{1}, \quad x=x_{2}, \quad u_{t}=u_{1}, \quad u_{x}=u_{2}
$$

can be written under the form of a first order system:

$$
\begin{aligned}
& \frac{\partial u_{2}}{\partial x_{1}}-\frac{\partial u_{1}}{\partial x_{2}}=0 \\
& \kappa_{1}\left(\frac{\partial u_{1}}{\partial x_{1}} \frac{\partial u_{2}}{\partial x_{2}}-\left(\frac{\partial u_{1}}{\partial x_{2}}\right)^{2}\right)+\kappa_{2} \frac{\partial u_{1}}{\partial x_{1}}+\kappa_{3} \frac{\partial u_{1}}{\partial x_{2}}+\kappa_{4} \frac{\partial u_{2}}{\partial x_{2}}+\kappa_{5}=0
\end{aligned}
$$

The admitted Lie symmetries of (129) are generated by the infinitesimal operator

$$
\Xi=\sum_{k=1}^{2} F_{k}\left(z_{1}, z_{2}\right) \Xi_{k}, \quad \Xi_{k}=\alpha_{k} \frac{\partial}{\partial x_{k}}+\frac{\partial}{\partial u_{k}}
$$

where $\alpha_{1}$ and $\alpha_{2}$ are (not both vanishing) constants such that

$$
\kappa_{1}+\kappa_{2} \alpha_{2}+\kappa_{4} \alpha_{1}+\kappa_{5} \alpha_{1} \alpha_{2}=0
$$

whereas

$$
z_{1}=x_{1}-\alpha_{1} u_{1}, \quad z_{2}=x_{2}-\alpha_{2} u_{2}
$$


and the functions $F_{1}\left(z_{1}, z_{2}\right)$ and $F_{2}\left(z_{1}, z_{2}\right)$ are solutions of the linear system

$$
\begin{aligned}
& \frac{\partial F_{2}}{\partial z_{1}}-\frac{\partial F_{1}}{\partial z_{2}}=0, \\
& \left(\kappa_{2}+\kappa_{5} \alpha_{1}\right) \frac{\partial F_{1}}{\partial z_{1}}+\kappa_{3} \frac{\partial F_{1}}{\partial z_{2}}+\left(\kappa_{4}+\kappa_{5} \alpha_{2}\right) \frac{\partial F_{2}}{\partial z_{2}}=0
\end{aligned}
$$

Theorem 11 can be used, whereupon, assuming that the dependent variables $u_{1}$ and $u_{2}$ depend upon the new independent variables $z_{1}$ and $z_{2}$, the system (129) reduces to the following linear form:

$$
\begin{aligned}
& \frac{\partial u_{2}}{\partial z_{1}}-\frac{\partial u_{1}}{\partial z_{2}}=0, \\
& \left(\kappa_{2}+\kappa_{5} \alpha_{1}\right) \frac{\partial u_{1}}{\partial z_{1}}+\kappa_{3} \frac{\partial u_{1}}{\partial z_{2}}+\left(\kappa_{4}+\kappa_{5} \alpha_{2}\right) \frac{\partial u_{2}}{\partial z_{2}}+\kappa_{5}=0
\end{aligned}
$$

Furthermore, the system (134) is equivalent to the linear second order partial differential equation

$$
\left(\kappa_{2}+\kappa_{5} \alpha_{1}\right) \frac{\partial^{2} u}{\partial z_{1}^{2}}+\kappa_{3} \frac{\partial^{2} u}{\partial z_{1} \partial z_{2}}+\left(\kappa_{4}+\kappa_{5} \alpha_{2}\right) \frac{\partial^{2} u}{\partial z_{2}^{2}}+\kappa_{5}=0
$$

\section{Reduction of First Order Quasilinear Systems to Homogeneous Form}

Another important application of Lie symmetries concerns the reduction of a first order quasilinear system of partial differential equations to a quasilinear system that is also autonomous and homogeneous.

In mathematical modelling of one-dimensional nonlinear wave propagation through non-dissipative and homogeneous media a relevant role is played by $2 \times 2$ quasilinear homogeneous and autonomous systems

$$
A^{0}(\mathbf{u}) \frac{\partial \mathbf{u}}{\partial t}+A^{1}(\mathbf{u}) \frac{\partial \mathbf{u}}{\partial x}=\mathbf{0}, \quad \mathbf{u}=\left[u_{1}, u_{2}\right]^{T}
$$

where $A^{0}$ and $A^{1}$ are $2 \times 2$ matrices depending on the unknown vector field $\mathbf{u}$, whereas $x$ and $t$ denote space and time coordinates, respectively. Of special relevance to wave propagation is the case when (136) is strictly hyperbolic, i.e., when $\operatorname{det}\left(A^{0}\right) \neq 0$ and the matrix $A^{1}$ admits two real distinct eigenvalues $\lambda, \mu$ (with respect to $A^{0}$ ) to which correspond two left eigenvectors $\mathbf{l}^{(\lambda)}, \mathbf{l}^{(\mu)}$ as well as two right eigenvectors $\mathbf{r}^{(\lambda)}, \mathbf{r}^{(\mu)}$ spanning the Euclidean space $E^{2}$.

In such a case, after the introduction of the Riemann invariants, several wave problems have been investigated, one of which being the solution of the Riemann problem where the solution connecting two different constant states is expressed in terms of centered waves admitted by (136) owing to the autonomous and homogeneous form.

For nonhomogeneous and dissipative media, the $2 \times 2$ quasilinear governing system which replaces (136) has the general form

$$
A^{0}(\mathbf{u}, x, t) \frac{\partial \mathbf{u}}{\partial t}+A^{1}(\mathbf{u}, x, t) \frac{\partial \mathbf{U}}{\partial x}=\mathbf{B}(\mathbf{u}, x, t)
$$

where $\mathbf{B}(\mathbf{u}, x, t)$ is a known column vector.

Models belonging to the class (137) arise from different physical contexts such as viscoelastic materials [65], nonlinear elastic rods with variable cross-section [66], nonlinear heat conduction [67], problems with cylindrical or spherical symmetry. 
The mathematical structure of the system (137) does not allow for the direct linearization via the hodograph transformation (always possible in the homogeneous and autonomous case) so that the determination of explicit solutions usually represents a difficult task.

As far as the hyperbolic wave propagation is concerned, in general the system (137) does not admit Riemann invariants along the characteristics, whereupon asymptotic methods are usually employed in order to get approximate simple wave solutions and to point out the main features of the wave interaction process.

In the last years various reduction methods have been developed, such as those based on hodograph-like or Backlünd transformations, allowing to map models belonging to the class (137) to the form $(136)[68,69]$. In particular, in [70] a direct method has been used for reducing to linear form $2 \times 2$ nonhomogeneous and nonautonomous first order quasilinear systems.

Nevertheless, the possibility of reducing (137) to autonomous and homogeneous form is strictly related to the symmetry properties of the model under investigation. In fact, necessary and sufficient conditions allowing for such a reduction are obtained; remarkably, when the approach here considered is applicable, it is possible to construct explicitly the map transforming nonhomogeneous and nonautonomous $2 \times 2$ quasilinear systems to homogeneous and autonomous form. The key idea is that any $2 \times 2$ homogeneous and autonomous first order quasilinear system is left invariant by an infinite-parameter Lie group of point transformations [11]. Therefore, if a nonhomogeneous and nonautonomous system can be reduced to homogeneous and autonomous form by an invertible point transformation, an infinite-parameter Lie group has to be admitted by the original system; conversely, if a nonhomogeneous and nonautonomous $2 \times 2$ quasilinear system admits a suitable infinite-parameter Lie group of point symmetries, then an invertible map exists transforming it to homogeneous and autonomous form [71].

Theorem 12 ([71]) The nonhomogeneous and nonautonomous $2 \times 2$ quasilinear system

$$
A^{0}(\mathbf{u}, x, t) \frac{\partial \mathbf{u}}{\partial t}+A^{1}(\mathbf{u}, x, t) \frac{\partial \mathbf{u}}{\partial x}=\mathbf{B}(\mathbf{u}, x, t)
$$

transforms, under the action of the one-to-one point variable transformation

$$
\xi=X(x, t), \quad \tau=T(x, t), \quad \mathbf{v}=\mathbf{V}(x, t, \mathbf{u})
$$

to the homogeneous and autonomous form, say

$$
\widehat{A}^{0}(\mathbf{v}) \frac{\partial \mathbf{v}}{\partial \tau}+\widehat{A}^{1}(\mathbf{v}) \frac{\partial \mathbf{v}}{\partial \xi}=\mathbf{0}, \quad \mathbf{v}=\left[v_{1}, v_{2}\right]^{T}
$$

if and only if the system (138) is left invariant by the Lie group of point symmetries

$$
\Xi=F_{1}(\mathbf{v}) \Xi_{1}+F_{2}(\mathbf{v}) \Xi_{2}
$$

where

$$
\Xi_{i}=\xi_{i} \frac{\partial}{\partial x}+\tau_{i} \frac{\partial}{\partial t}+\eta_{i}^{(1)} \frac{\partial}{\partial u_{1}}+\eta_{i}^{(2)} \frac{\partial}{\partial u_{2}} \quad(i=1,2)
$$


are commuting infinitesimal operator, i.e., $\left[\Xi_{1}, \Xi_{2}\right]=0$, the infinitesimal generators $\xi_{i}, \tau_{i}, \eta_{i}^{(1)}$ and $\eta_{i}^{(2)}$ may depend on $x, t, u_{1}$ and $u_{2}$, whereas $F_{1}(\mathbf{v})$ and $F_{2}(\mathbf{v})$ are solutions of the linear system of partial differential equations

$$
\widehat{A}^{1}(\mathbf{v}) J \nabla_{\mathbf{v}} F_{1}(\mathbf{v})-\widehat{A}^{0}(\mathbf{v}) J \nabla_{\mathbf{v}} F_{2}(\mathbf{v})=\mathbf{0}, \quad J=\left[\begin{array}{cc}
0 & 1 \\
-1 & 0
\end{array}\right]
$$

where $\nabla_{\mathbf{v}}$ is the gradient operator with respect to the components of the indicated subscript, and it is

$$
\Xi_{1} X=0, \quad \Xi_{1} T=1, \quad \Xi_{2} T=0, \quad \Xi_{2} X=1, \quad \Xi_{1} \mathbf{v}=\Xi_{2} \mathbf{v}=0
$$

See [71].

It is worth of underlining that the reduction of $2 \times 2$ quasilinear first order systems of partial differential equations to homogeneous and autonomous form is intimately related to the possibility of their transformation to linear form. For general first order quasilinear systems involving more than two independent variables and/or more than two dependent variables, this link can not be invoked. Nevertheless, also in this case it is possible to recover the necessary and sufficient conditions allowing for the transformation to homogeneous and autonomous form within the framework of Lie group analysis.

Given the first order quasilinear system

$$
\sum_{\alpha=0}^{n} A^{\alpha}(\mathbf{u}, \mathbf{x}) \frac{\partial \mathbf{u}}{\partial x_{\alpha}}=\mathbf{B}(\mathbf{u}, \mathbf{x})
$$

where $x_{0}=t$ is the time, $x_{1}, \ldots, x_{n}$ the space variables, $u \in \mathbb{R}^{m}, A^{\alpha} m \times m$ matrices ( $m$ and $n$ arbitrary), an invertible mapping of the form

$$
\mathbf{y}=\mathbf{Y}(\mathbf{x}), \quad \mathbf{v}=\mathbf{V}(\mathbf{u}, \mathbf{x})
$$

such that (144) is transformed to

$$
\sum_{\alpha=0}^{n} \widetilde{A}^{\alpha}(\mathbf{v}) \frac{\partial \mathbf{v}}{\partial y_{\alpha}}=\mathbf{0}
$$

exists if and only if some Lie point symmetries with a suitable Lie algebraic structure are admitted by (144).

Some considerations are in order. Every system of the form (146) is invariant with respect to the $(n+1)$ translations of the independent variables and with respect to a uniform scaling of the $y_{\alpha}$, i.e., every system of the form (146) admits the Lie symmetries generated by

$$
\Xi_{\alpha}=\frac{\partial}{\partial y_{\alpha}} \quad(\alpha=0,1, \ldots, n), \quad \Xi_{n+1}=\sum_{\alpha=0}^{n} y_{\alpha} \frac{\partial}{\partial y_{\alpha}}
$$

spanning an $(n+2)$-dimensional solvable Lie algebra where the only non-zero commutators are

$$
\left[\Xi_{\alpha}, \Xi_{n+1}\right]=\Xi_{\alpha}, \quad(\alpha=0,1, \ldots, n)
$$

Moreover, systems of the form (144) are mapped to first order quasilinear systems by an invertible transformation like (145), i.e., the transformations (145) preserve the quasilinear structure. Finally, the structure of the Lie algebra of point symmetries of a system of partial differential equations is not changed by an invertible point transformation [10,11] All these considerations allow us to prove the following theorem. 
Theorem 13 ([72]) A nonhomogeneous and/or nonautonomous first order quasilinear system of the form (144) can be transformed by the invertible map like (145) into an autonomous and homogeneous first order quasilinear system if and only if it admits as subalgebra of its Lie point symmetries an $(n+2)$-dimensional Lie algebra spanned by the vector fields

$$
\begin{aligned}
& \Xi_{\alpha}=\sum_{\beta=0}^{n} \xi_{\alpha}^{\beta} \frac{\partial}{\partial x_{\beta}}+\sum_{A=1}^{m} \eta_{\alpha}^{A} \frac{\partial}{\partial u^{A}}, \quad(\alpha=0, \ldots, n) \\
& \Xi_{n+1}=\sum_{\beta=0}^{n} \xi_{n+1}^{\beta} \frac{\partial}{\partial x_{\beta}}+\sum_{A=1}^{m} \eta_{n+1}^{A} \frac{\partial}{\partial u_{A}} \\
& {\left[\Xi_{\alpha}, \Xi_{\beta}\right]=0, \quad\left[\Xi_{\alpha}, \Xi_{n+1}\right]=\Xi_{\alpha}, \quad \alpha, \beta=0,1, \ldots, n}
\end{aligned}
$$

Furthermore, it has to be

$$
\operatorname{rank}\left\|\xi_{\alpha}^{\beta}\right\|=n+1 \quad(\alpha=0,1, \ldots, n ; \beta=0,1, \ldots, n+2)
$$

$\mathbf{y}$ and $\mathbf{v}$ are the canonical variables of the vector fields $\Xi_{\alpha}(\alpha=0,1, \ldots, n), \mathbf{v}$ are invariants of all $(n+2)$ generators, and it is $\Xi_{n+1} y_{\alpha}=y_{\alpha}(\alpha=0,1, \ldots, n)$.

Application of theorem 13 requires the following steps.

1. determine the Lie algebra $L$ of point symmetries of system (144);

2. if $\operatorname{dim}(L) \geq n+2$ :

- determine the $(n+2)$-dimensional Lie subalgebras (an optimal system suffices), for instance by means of the Mathematica ${ }^{\circledR}$ package SymboLie [40];

- check if among the Lie subalgebras there is a Lie algebra having the required structure;

- find the canonical variables of the symmetries, and reduce the system to homogeneous and autonomous form.

Of course, theorem 13 can be applied also to $2 \times 2$ systems.

\subsection{Reduction to Homogeneous Form: Applications}

Let us give two simple applications of theorem 13.

Example 12 (Nonlinear Hyperbolic Heat Equation) Let us consider the hyperbolic heat equation proposed in [67]

$$
\frac{\partial}{\partial t}\left(\frac{\partial u}{\partial t}+\frac{u}{\tau_{0}}\right)-\frac{\partial}{\partial x}\left(\frac{\chi_{0}^{2}}{u^{2}} \frac{\partial u}{\partial x}\right)=0
$$

where $\chi_{0}$ and $\tau_{0}$ are positive constants; this equation has been reduced [67] to the standard linear telegraph equation by means of a reciprocal Backlünd transformation. Furthermore, by setting $u=1 / \bar{u}$ and choosing $\tau_{0}=\chi_{0}=1$, the equation (149) specializes to the nonlinear telegraph equation linearized 
in [11] within the framework of potential symmetries. Actually, by setting $u=u_{1}$ and introducing the potential function $u_{2}(x, t)$, we are led to the following $2 \times 2$ quasilinear system

$$
\begin{aligned}
& \frac{\partial u_{1}}{\partial t}-\frac{\partial u_{2}}{\partial x}=-\frac{u_{1}}{\tau_{0}} \\
& \frac{\partial u_{2}}{\partial t}-\frac{\chi_{0}^{2}}{u_{1}^{2}} \frac{\partial u_{1}}{\partial x}=0
\end{aligned}
$$

The system (150) admits the Lie point symmetries generated by the infinitesimal operators

$$
\begin{aligned}
& \Xi_{1}=\frac{\partial}{\partial x} \\
& \Xi_{2}=\exp \left(-t / \tau_{0}\right)\left(\tau_{0} \frac{\partial}{\partial t}-u_{1} \frac{\partial}{\partial u_{1}}\right) \\
& \Xi_{3}=x \frac{\partial}{\partial x}+\tau_{0} \frac{\partial}{\partial t}-u_{1} \frac{\partial}{\partial u_{1}}
\end{aligned}
$$

Using theorem 13, we introduce the new variables

$$
\begin{aligned}
& \xi=x, \quad \tau=\exp \left(\frac{t}{\tau_{0}}\right) \\
& v_{1}=\exp \left(\frac{t}{\tau_{0}}\right) u_{1}, \quad v_{2}=u_{2}
\end{aligned}
$$

and the system (150) reduces to the form

$$
\begin{aligned}
& \frac{\partial v_{1}}{\partial \tau}-\tau_{0} \frac{\partial v_{2}}{\partial \xi}=0 \\
& \frac{\partial v_{2}}{\partial \tau}-\frac{\chi_{0}^{2} \tau_{0}}{v_{1}^{2}} \frac{\partial v_{1}}{\partial \xi}=0
\end{aligned}
$$

i.e., it results an autonomous and homogeneous system.

Example 13 (Rate-Type Materials) The equations

$$
\begin{aligned}
& \frac{\partial u_{1}}{\partial t}-\frac{\partial u_{2}}{\partial x}=0 \\
& \frac{\partial u_{2}}{\partial t}-\phi\left(t, u_{2}\right) \frac{\partial u_{1}}{\partial x}=\psi\left(t, u_{2}\right) \\
& \phi\left(t, u_{2}\right)=\varphi\left(\exp (-t) u_{2}\right), \quad \psi\left(t, u_{2}\right)=u_{2}-\varphi\left(\exp (-t) u_{2}\right)
\end{aligned}
$$

where $u_{1}$ denotes the Lagrangian velocity and $u_{2}$ the stress, belonging to a class of equations introduced in [65] (see also [68]) to describe the experimental behaviour of rate-type materials undergoing loading-unloading one-dimensional processes, admits the Lie point symmetries generated by

$$
\begin{aligned}
& \Xi_{1}=\exp (-t)\left(\frac{\partial}{\partial t}+\exp (-t) u_{2} \frac{\partial}{\partial u_{2}}\right) \\
& \Xi_{2}=\frac{\partial}{\partial x}+\frac{\partial}{\partial u_{1}} \\
& \Xi_{3}=x \frac{\partial}{\partial x}+\frac{\partial}{\partial t}+x \frac{\partial}{\partial u_{1}}+\exp (-t) u_{2} \frac{\partial}{\partial u_{2}}
\end{aligned}
$$


Also in this case, theorem 13 can be applied, and, through the introduction of the variable transformation

$$
\begin{aligned}
& \xi=x, \quad \tau=\exp (t) \\
& v_{1}=u_{1}-x, \quad v_{2}=u_{2} \exp (-t)
\end{aligned}
$$

we are able to recover the system

$$
\begin{aligned}
& \frac{\partial v_{1}}{\partial \tau}-\frac{\partial v_{2}}{\partial \xi}=0 \\
& \frac{\partial v_{2}}{\partial \tau}-\varphi\left(v_{2}\right) \frac{\partial v_{1}}{\partial \xi}=0
\end{aligned}
$$

which is autonomous and homogeneous.

The reduction of a nonhomogeneous quasilinear system to homogeneous form, besides its intrinsic interest, may reveal useful in investigating a well known problem connected with a system of hyperbolic conservation laws, say the Riemann problem $[73,74]$, where one takes a piecewise constant initial datum with a single discontinuity. As well known, there is an existence and uniqueness theorem for the Riemann problem for a system of conservation laws; on the contrary, analogous results have not been obtained yet for a system of balance laws, even for a generalized (i.e., piecewise non-constant initial data) Riemann problem [75].

In the case in which we have a system of balance laws and the application of theorem 13 leads to a system of conservation laws, then one may investigate a Riemann problem (classical or generalized) for the original system of balance laws by studying an associated Riemann problem (which can be classical or generalized) for a system of conservation laws [72]. Once the latter problem has been solved, thanks to the inverse transformation, it is possible to obtain the corresponding solution of the original system. Such an approach has been used in [76] for the system (154).

\section{Nonlocal Symmetries}

In the preceeding sections continuous symmetries of local type of differential equations have been mainly considered. In general, a symmetry of a differential equation is any transformation of its solution manifold into itself, i.e., a symmetry maps any solution to another solution of the same equation. Hence, continuous symmetry transformations (which are essentially deformations of solutions) are defined topologically and thus are not restricted to local transformations acting on the space of independent and dependent variables and their derivatives. In this sense, any differential equation possesses symmetries.

Nevertheless, examples of ordinary differential equations, which can be solved by quadrature and do not possess Lie symmetries, are known in literature [32,77]. Their investigation, joined to the observation that in certain cases hidden symmetries [77-79] appear when the order of a differential equation is decreased or increased, led in the last twenty years to many research papers devoted to the investigation of nonlocal symmetries, i.e., symmetries with one or more of the coefficients containing an integral (see [32] for a sketch of the evolution of the subject of nonlocal symmetries, especially centered on nonlocal symmetries of ordinary differential equations). Nonlocal symmetries exist also for partial differential equations [19], and may be used for instance to generate nonlocal conservation laws [19,80], thus extending Noether's theorem. 
For ordinary differential equations, many different examples and occurrences of nonlocal symmetries (called hidden symmetries) were reported in the early Nineties of last century [77-79] (see also [32] and the references therein quoted). First, it was observed that there could be gain of symmetry in the reduction of order of an ordinary differential equation, subsequently, the reverse process was also observed, gain of symmetry when the order of an equation is increased. Hidden symmetries of Type I arise when the order of an equation is increased, and Type II when the order of an equation is decreased.

Example 14 [32] Consider the fourth order ordinary differential equation

$$
2 u u^{\prime \prime \prime \prime}+5 u^{\prime} u^{\prime \prime \prime}=0, \quad\left({ }^{\prime}=\frac{d}{d x}\right)
$$

arising in the study of the symmetries of the Emden-Fowler equation [81]. Equation (158) possesses three Lie point symmetries generated by

$$
\Xi_{1}=\frac{\partial}{\partial x}, \quad \Xi_{2}=x \frac{\partial}{\partial x}, \quad \Xi_{3}=u \frac{\partial}{\partial u}
$$

Use of symmetry $\Xi_{1}$, through the introduction of the invariants $t=u, v=u^{\prime}$, yields

$$
2 t\left(u^{2} u^{\prime \prime \prime}+4 u u^{\prime} u^{\prime \prime} u u^{\prime 3}\right)+5\left(u^{2} u^{\prime \prime}+u u^{\prime 2}=0, \quad\left({ }^{\prime}=\frac{d}{d t}\right)\right.
$$

The latter equation inherits the symmetries $\Xi_{2}$ and $\Xi_{3}$ that read as

$$
\widetilde{\Xi}_{2}=v \frac{\partial}{\partial v}, \quad \widetilde{\Xi}_{3}=t \frac{\partial}{\partial t}+v \frac{\partial}{\partial v}
$$

Using these inherited symmetries in the process of order reduction one arrives at a very complicated Abel's equation of the second kind.

However, if one computes the point symmetries of (160) one is able to recover also the symmetry

$$
\widetilde{\Xi}_{4}=2 t^{2} \frac{\partial}{\partial t}+t v \frac{\partial}{\partial v}
$$

Remarkably, using this symmetry, whose invariants are

$$
r=v t^{-1 / 2}, \quad s=\frac{1}{2}\left(v^{\prime} t^{3 / 2}-\frac{1}{2} v t^{1 / 2}\right)^{2}
$$

the reduced equation,

$$
s^{\prime \prime}+3 s^{\prime}+2 s=0, \quad\left({ }^{\prime}=\frac{d}{d r}\right)
$$

is linear.

It is evident that the symmetry $\widetilde{\Xi}_{4}$ can not come from a point symmetry of (158). Nevertheless, by requiring that $\widetilde{\Xi}_{4}$ has its origin in a symmetry of (158), this symmetry must have the form

$$
\Xi_{4}=3\left(\int u d x\right) \frac{\partial}{\partial x}+2 u^{2} \frac{\partial}{\partial u}
$$

i.e., it is a nonlocal symmetry. This is referred to as hidden symmetry of Type II since it appears as a point symmetry on reduction of order. 
Nonlocal symmetries emerge also when differential equations do not possess Lie point symmetries and nevertheless may be integrated.

Example 15 ([32]) Consider the second order ordinary differential equations

$$
u^{\prime \prime}=\frac{u^{\prime 2}}{u}+f^{\prime}(x) u^{p+1}+p f(x) u^{p} u^{\prime}
$$

which does not admit Lie point symmetries for general $f(x)$ and $p$, and nevertheless is trivially integrable; in fact, through the nonlocal change of variables

$$
u=-\frac{y^{\prime}}{p f(x) w}, \quad W=\log w^{\prime}
$$

becomes the linear equation

$$
W^{\prime \prime}=0
$$

possessing eight point symmetries spanning the projective algebra in the plane. These symmetries of course correspond to very complicated nonlocal symmetries of (166).

At the beginning nonlocal symmetries appeared to be related to special and artificious problems, or to counterexamples where Lie group theory seemed unfruitful. In this context we may quote also the introduction of $\lambda$-symmetries [82-84] and $\mu$-symmetries [85,86], that may be interpreted as nonlocal symmetries too [87].

In the last years the importance of nonlocal symmetries grew constantly and various contexts make use of them. For instance, the notion of complete symmetry group [88,89], introduced to specify completely a differential equation by means of its symmetries, often requires the use of nonlocal symmetries. In [90] it has been shown how to derive the complete symmetry group for the Kepler problem, as introduced in [88], within the framework of Lie group analysis. Also, the notion of complete symmetry group has been extended to partial differential equations [91].

Despite their abundance, nonlocal symmetries have the disadvantage that there is not a direct approach for their complete determination. However, it is possible to determine systematically nonlocal symmetries of differential equations by applying suitably the Lie's algorithm for local symmetries to a nonlocally related system of differential equations [19].

This systematic approach for finding nonlocal symmetries of partial differential equations [19] makes use of the existence of local conservation laws to introduce a nonlocally related system of differential equations. Alternatively, a nonlocally related system of differential equations for a given source system may be obtained considering subsystems of the given system of differential equations, by excluding one of the dependent variables, or interchanging one or more independent and dependent variables.

Bluman and coworkers [11,19] investigated largely the so called potential symmetries (which are nonlocal symmetries) by realizing such symmetries as local symmetries which are admitted by an associated auxiliary system (potential system) of differential equations. For a system of partial differential equations

$$
\Delta\left(\mathbf{x}, \mathbf{u}, \mathbf{u}^{(1)}, \ldots, \mathbf{u}^{(k)}\right) \equiv \Delta[\mathbf{x}, \mathbf{u}]=\mathbf{0}
$$

when at least one equation of the system can be written in a conserved form with respect to some choice of its variables, it is possible to introduce auxiliary dependent variables $\mathbf{v}$, which are potentials, and 
an auxiliary system of partial differential equations $\widetilde{\Delta}[\mathbf{x}, \mathbf{u}, \mathbf{v}]=\mathbf{0}$ embedding $\Delta[\mathbf{x}, \mathbf{u}]=\mathbf{0}$. Any solution $(\mathbf{u}(\mathbf{x}), \mathbf{v}(\mathbf{x}))$ of $\widetilde{\Delta}[\mathbf{x}, \mathbf{u}, \mathbf{v}]=\mathbf{0}$ will define a solution $\mathbf{u}(\mathbf{x})$ of $\boldsymbol{\Delta}[\mathbf{x}, \mathbf{u}]=\mathbf{0}$, and to any solution $\mathbf{u}(\mathbf{x})$ of $\Delta[\mathbf{x}, \mathbf{u}]=\mathbf{0}$ there corresponds a function $\mathbf{v}(\mathbf{x})$ such that $(\mathbf{u}(\mathbf{x}), \mathbf{v}(\mathbf{x}))$ defines a solution of $\widetilde{\Delta}[\mathbf{x}, \mathbf{u}, \mathbf{v}]=\mathbf{0}$.

Local symmetries admitted by $\widetilde{\Delta}[\mathbf{x}, \mathbf{u}, \mathbf{v}]=\mathbf{0}$ map any solution into another solution of $\widetilde{\Delta}[\mathbf{x}, \mathbf{u}, \mathbf{v}]=\mathbf{0}$, and hence map any solution of $\Delta[\mathbf{x}, \mathbf{u}]=\mathbf{0}$ into another solution of $\Delta[\mathbf{x}, \mathbf{u}]=\mathbf{0}$. As a consequence, local symmetries of $\widetilde{\Delta}[\mathbf{x}, \mathbf{u}, \mathbf{v}]=\mathbf{0}$ will induce nonlocal symmetries of $\Delta[\mathbf{x}, \mathbf{u}]=\mathbf{0}$ if the infinitesimals depend explicitly on the potential variables v. Such nonlocal symmetries are called potential symmetries of $\Delta[\mathbf{x}, \mathbf{u}]=\mathbf{0}$, and, remarkably, can be computed by Lie's algorithm since they are realized as local symmetries admitted by the auxiliary system $\widetilde{\Delta}[\mathbf{x}, \mathbf{u}, \mathbf{v}]=\mathbf{0}$. In passing we note that, in the Example 10, the system (118) is a potential system of equation (117), and the local point symmetries (119) of system (118) are indeed potential symmetries, and hence nonlocal symmetries, of equation (117).

The construction of potential systems may be extended by considering trees of nonlocally related systems [19]. Within each tree, all systems will be equivalent in the sense that the solution set of any system in a tree can be obtained from the solution set of any other system in the same tree. Consider a given system $\boldsymbol{\Delta}[x, t, \mathbf{u}]=\mathbf{0}$ involving two independent variables $x$ and $t$, having $n$ known local conservation laws and $n$ corresponding potential systems denoted by $\widetilde{\boldsymbol{\Delta}}_{i}\left[x, t, \mathbf{u}, \mathbf{v}_{i}\right]=\mathbf{0}(i=1, \ldots, n)$.

This set of $n$ nonlocally related systems for the given system $\Delta[x, t, \mathbf{u}]=\mathbf{0}$ may be extended considering potential systems for each of the potential systems, obtained from the conservation laws of the latter to introduce further potential variables: in principle, one can obtain up to $2^{n}-1$ distinct nonlocally related systems from the $n$ local conservation laws. A procedure for constructing a hierarchy (tree) of nonlocally related potential systems and subsystems for a given system of partial differential equations is fully described in [19] (see also the references therein quoted). The procedure requires at the various steps the elimination of locally related systems.

In dealing with systems of partial differential equations involving more than two independent variables the situation becomes more complex. In fact, in order to obtain interesting potential systems from a local conservation law, it is necessary to introduce gauge constraints that relate the potential variables resulting from local conservation laws. The interested reader is referred to [19] for the details as well as for the application of the procedure to physically relevant systems of partial differential equations (nonlinear diffusion equations, nonlinear wave equations, planar gas dynamics equations). Nonlocal symmetries in turn provide useful in obtaining nonlocal conservation laws, as well as building non-invertible mappings between different systems of partial differential equations.

\section{Conclusions}

Various results, either classical and well established or recent, concerning the application of Lie symmetries to differential equations, have been presented.

A lot of interesting and very promising results have not been considered, such as:

- the equivalence transformations $[7,31,92]$ that are continuous transformations mapping a specific class of differential equations involving arbitrary functions or parameters into the same class, and 
therefore are more general than symmetries (i.e., an equation belonging to a class is mapped to another equation of the same class); equivalence transformations provide useful when group classification of differential equations containing arbitrary functions is needed [93] and also to linearize differential equations;

- the approximate Lie symmetries [29,30], useful to apply suitably Lie's machinery to differential equations containing small terms; approximate Lie symmetries allow to build approximate invariant solutions to partial differential equations, or approximate reduction of order of ordinary differential equations, or approximate conservation laws.

Roughly speaking, all the problems we faced throughout this paper fall into the general class of the so called direct Lie problems [59], in the sense that the main aim is to find the symmetries admitted by a given differential equation (or by a class of differential equations) and then use the symmetries to investigate the properties of the solutions.

To the problem of finding symmetries of differential equations a natural inverse problem can be associated, namely, the problem of finding the most general form of a differential equations admitting a given Lie algebra of infinitesimal point symmetries. The inverse problem has been considered for the first time in [8] from an algorithmic viewpoint in order to characterize all differential equations admitting a given group. An interesting contribution has been given by Rosenhaus in 1982 [94] (see also [95,96]), who posed the problem of the unique determination of a differential equations by its group; in fact, in [94] the author considered the projective algebra of $\mathbb{R}^{3}$ and its subalgebras, and was able to prove that the equation of vanishing Gaussian curvature of surfaces in $\mathbb{R}^{3}$ (which is a Monge-Ampère-type equation) is uniquely determined by its Lie point symmetries. Differential equations uniquely determined by their Lie point symmetries, called Lie remarkable equations [97-99], have been introduced and investigated in a series of recent papers; the notion of Lie remarkable equations, in turn, is connected with the notion of complete symmetry groups [88,89], even if the latter approach makes use of nonlocal symmetries.

\section{Acknowledgments}

Work supported by "Gruppo Nazionale per la Fisica Matematica" of the "Istituto Nazionale di Alta Matematica Francesco Severi” and by Research Grants of the University of Messina.

The author dedicates this paper to the memory of Andrea Donato, unforgettable friend and mentor, passed away ten years ago, who enthusiastically addressed him to the study of Lie symmetries of differential equations.

The anonymous referees, whose comments and suggestions helped to improve the paper, are gratefully acknowledged.

\section{References}

1. Noether, E. Invariante Variationsprobleme. Nachr. v. d. Ges. d. Wiss. zu Göttingen, Math. Phys. K1. 1918, 235-257; English translation, Transp. Th. Stat. Phys. 1971, 1, 186-207.

2. Yaglom, I.M. Felix Klein and Sophus Lie: Evolution of the Idea of Symmetry in the Nineteenth Century; Birkhäuser: Boston, MA, USA, 1988. 
3. Lie, S.; Engel, F. Theorie der transformationsgruppen; Teubner: Leipzig, Germany, 1888.

4. Lie, S. Vorlesungen über differentialgleichungen mit bekannten infinitesimalen transformationen; Teubner: Leipzig, Germany, 1891.

5. Birkhoff, G. Hydrodynamics -A study in Logic, Fact and Similitude; Princeton University Press: Princeton, NJ, USA, 1950.

6. Sedov, L.I. Similarity and Dimensional Methods in Mechanics; Academic Press: New York, NY, USA, 1959.

7. Ovsiannikov, L.V. Group Analysis of Differential Equations; Academic Press: New York, NY, USA, 1982.

8. Bluman, G.W.; Cole, J.D. Similarity Methods for Differential Equations; Springer-Verlag: New York, NY, USA, 1974.

9. Ibragimov, N.H. Transformation Groups Applied to Mathematical Physics; Reidel Publishing Company: Dordrecht, the Netherlands, 1985.

10. Olver, P.J. Applications of Lie Groups to Differential Equations; Springer: New York, NY, USA, 1986.

11. Bluman, G.W.; Kumei, S. Symmetries and Differential Equations; Springer-Verlag: New York, NY, USA, 1989.

12. Stephani, H. Differential Equations. Their Solutions Using Symmetries; Cambridge University Press: Cambridge, UK, 1989.

13. Ibragimov, N.H. Handbook of Lie Group Analysis of Differential Equations; CRC Press: Boca Raton, FL, USA, 1994, 1995, 1996; volumes 3.

14. Olver, P.J. Equivalence, Invariants, and Symmetry; Cambridge University Press: Cambridge, UK, 1995.

15. Baumann, G. Symmetry Analysis of Differential Equations with Mathematica; Springer: New York, NY, USA, 2000.

16. Cantwell, B.J. Introduction to Symmetry Analysis; Cambridge University Press: Cambridge, UK, 2002.

17. Bluman, G.W.; Anco, S.C. Symmetry and Integration Methods for Differential Equations; Springer: New York, NY, USA, 2002,

18. Meleshko, S.V. Methods for Constructing Exact Solutions of Partial Differential Equations; Springer: New York, NY, USA, 2005.

19. Bluman, G.W.; Cheviakov, A.F.; Anco, S.C. Applications of Symmetry Methods to Partial Differential Equations; Springer: New York, NY, USA, 2009.

20. Schwarz, F. Automatically determining symmetries of partial differential equations. Computing 1985, 34, 91-106; Addendum. Computing 1986, 36, 279-280.

21. Schwarz, F. Symmetries of differential equations from Sophus Lie to computer algebra. Siam Rev. 1988, 30, 450-481.

22. Sherring, J.; Prince, G. DIMSYM-Symmetry determination and linear differential equations package. Technical Report: Department of Mathematics, LaTrobe University, Bundoora, Australia, January 1996. 
23. Hereman, W. Review of symbolic software for the computation of Lie symmetries of differential equations. Euromath Bull. 1994, 1, 45-82.

24. Oliveri, F. Relie: A Reduce program for calculating Lie symmetries. Technical Report: Department of Mathematics, University of Messina, Italy, November 2001. Available online: http://mat520.unime.it/oliveri (accessed December 20, 2009)

25. Bluman, G.W.; Cole, J.D. The general similarity solution of the heat equation. J. Math. Mech. 1969, 18, 1025-1042.

26. Olver, P.J.; Rosenau, P. The construction of special solutions to partial differential equations. Phys. Lett. A 1986, 114, 107-112.

27. Olver, P.J.; Rosenau, P. Group-invariant solutions of differential equations. SIAM J. Appl. Math. 1987, 47, 263-278.

28. Pucci, E.; Saccomandi G. On the weak symmetry groups of partial differential equations. J. Math. Anal. Appl. 1992, 163, 588-598.

29. Baikov, V.A.; Gazizov, R.K.; Ibragimov, N.H. Approximate symmetries of equations with a small parameter. Math. U.S.S.R.-Sb. 1989, 64, 427-441.

30. Ibragimov, N.H.; Kovalev, V.F. Approximate and Renormgroup Symmetries; Beijing and Springer-Verlag: Berlin, Germany, 2009.

31. Lisle, I.G. Equivalence transformations for classes of differential equations. Ph.D. Thesis, University of British Columbia, Canada, 1992.

32. Leach, P.G.L.; Andriopoulos, K. Nonlocal symmetries: Past, present and future. Appl. Anal. Discrete Math. 2007, 1, 150-171.

33. Humphreys, J.E. Introduction to Lie Algebras and Representation Theory; Springer-Verlag: New York, NY, USA, 1972.

34. Erdmann, K.; Wildon M.J. Introduction to Lie Algebras; Springer: New York, NY, USA, 2006.

35. Bianchi, L. Lezioni sulla teoria dei gruppi continui finiti di trasformazione; Enrico Spoerri Editore: Pisa, Italy, 1918.

36. Bluman, G. A reduction algorithm for an ordinary differential equations admitting a solvable Lie group. SIAM J. Appl. Math. 1990, 50, 1689-1705.

37. Ibragimov, N.H. Group analysis of ordinary differential equations and the invariance principle in mathematical physics. Russ. Math. Surv. 1992, 47, 89-156.

38. Fels, M.E. Integrating scalar ordinary differential equations with symmetry revisited. Found. Comput. Math. 2007, 7, 417-454.

39. Ovsiannikov, L.V. The PODMODELI program. Gas dynamics. Prikl. Mat. Mekh. 1994, 58, 30-55.

40. Margheriti, L.; Oliveri, F. A Mathematica package for obtaining the optimal system of subalgebras of a Lie algebra. Preprint, 2008, 1-21.

41. Hairer, E.; Lubich, Ch.; Wanner, G. Geometric Numerical Integration; Springer: New York, NY, USA, 2002.

42. Iserles, A.; Munthe-Kaas, H.Z.; Nørsett, S.P.; Zanna, A. Lie-group methods. Acta Numer. 2000, 9 , 215-365.

43. Boyer, T.H. Continuous symmetries and conserved currents. Ann. Phys. 1967, 42, 445-466. 
44. Anco, S.C.; Bluman, G.W. Direct construction method for conservation laws of partial differential equations. Part I: Examples of conservation law classifications. Eur. J. Appl. Math. 2002, 13, $545-566$.

45. Anco, S.C.; Bluman, G.W. Direct construction method for conservation laws of partial differential equations. Part II: General treatment. Eur. J. Appl. Math. 2002, 13, 567-585.

46. Donato, A.; Oliveri, F. When nonautonomous equations are equivalent to autonomous ones. Appl. Anal. 1995, 58, 313-323.

47. Donato, A.; Oliveri, F. How to build up variable transformations allowing one to map nonlinear hyperbolic equations into autonomous or linear ones. Transp. Th. Stat. Phys. 1996, 25, 303-322.

48. Boillat, G. La propagation des ondes; Gauthier-Villars: Paris, France, 1974.

49. Donato, A. Similarity analysis and non-linear wave propagation. Int. J. Non-linear Mech. 1987, 22, 307-314.

50. Ames, W.F.; Donato, A. On the evolution of weak discontinuities in a state characterized by invariant solutions. Int. J. Non-linear Mech. 1988, 23, 167-174.

51. Donato, A.; Ruggeri, T. Similarity solutions and strong shocks in extended thermodynamics of rarefied gas. J. Math. Anal. Appl. 2000, 251, 395-405.

52. Müller, I.; Ruggeri, T. Rational Extended Thermodynamics; Springer-Verlag: New York, NY, USA, 1998.

53. Donato, A.; Oliveri, F. Reduction to autonomous form by group analysis and exact solutions of axi-symmetric MHD equations. Math. Comput. Model. 1993, 18, 83-90.

54. Oliveri, F.; Speciale, M.P. Exact solutions to the unsteady equations of perfect gases through Lie group analysis and substitution principles. Int. J. Non-linear Mech. 2002, 37, 257-274.

55. Oliveri, F.; Speciale, M.P. Exact solutions to the ideal magneto-gas-dynamics equations through Lie group analysis and substitution principles. J. Phys. A-Math. Gen. 2005, 38, 8803-8820.

56. Sharma, V.D.; Radha, R. Exact solutions of Euler equations of ideal gasdynamics via Lie group analysis. ZAMP 2007, 59, 1029-1038.

57. Hirota, R. Exact solutions of the equation describing cylindrical solitons. Phys. Lett. A 1979, 71, 393-394.

58. Grad, H. Principles of the kinetic theory of gases. In Handbuch der Physik; Springer: Berlin, Germany, 1958; pp. 205-294.

59. Oliveri, F. Lie symmetries of differential equations: direct and inverse problems. Note Matematica 2005, 23, 195-216.

60. Kumei, S.; Bluman, G.W. When nonlinear differential equations are equivalent to linear differential equations. SIAM J. Appl. Math. 1982, 42, 1157-1173.

61. Donato, A.; Oliveri, F. Linearization procedure of nonlinear first order systems of PDE's by means of canonical variables related to Lie groups of point transformations. J. Math. Anal. Appl. 1994, 188, 552-568.

62. Müller, E.A.; Matschat, K. Uber das Auffinden von Ahnlichkeitslösungen partieller Differentialgleichungssysteme unter Benutzung von Transformationsgruppen, mit Anwendungen auf Probleme der Stromungsphysik. In Miszellaneen der Angewandten Mechanik; Akademie-Verlag: Berlin, Germany, 1962; pp. 190-222. 
63. Fokas, A.S.; Yortsov, Y.C. On the exact solvable equation $S_{t}=\left[(\beta S+\gamma)^{-2} S_{x}\right]_{x}+(\beta S+\gamma)^{-2} S_{x}$ occurring in two-phase flow in porous media. SIAM J. Appl. Math. 1982, 42, 318-332.

64. Oliveri, F. Linearizable second order Monge-Ampère equations. J. Math. Anal. Appl. 1998, 218, 329-345.

65. Cristescu, N. Dynamic Plasticity; J. Wiley \& Sons: New York, NY, USA, 1964.

66. Jeffrey, A. Acceleration wave propagation in hyperelastic rods of variable cross-section. Wave Motion 1982, 4, 173-180.

67. Rogers, C.; Ruggeri, T. A reciprocal Bäcklund transformation: application to a non-linear hyperbolic model in heat conduction. Lett. Nuovo Cimento 1985, 2, 289-296.

68. Varley, E.; Seymour, B. Exact solutions for large amplitude waves in dispersive and dissipative systems. Stud. Appl. Math. 1985, 72, 241-262.

69. Fusco, D.; Manganaro, N. A class of linearizable models and generation of material response functions to nonlinear hyperbolic heat conduction. J. Math. Phys. 1991, 32, 3043-3046.

70. Currò, C.; Valenti, G. A linearization procedure for quasilinear nonhomogeneous and nonautonomous $2 \times 2$ first-order systems. Int. J. Non-Linear Mech. 1996, 31, 377-386.

71. Currò, C.; Oliveri, F. Reduction of nonhomogeneous quasilinear $2 \times 2$ systems to homogeneous and autonomous form. J. Math. Phys. 2008, 49, 103504-1-103504-11.

72. Oliveri, F. Lie symmetries and systems of balance laws. In Proceedings "Wascom 2009", 15th Conference on Waves and Stability in Continuous Media, Mondello (Palermo), Italy, June 27 - July 1, 2009; World Scientific: Singapore, 2010 (to appear).

73. Smoller, J. Shock Waves and Reaction-Diffusion Equations; Springer-Verlag: New York, NY, USA, 1983.

74. Dafermos, C.M. Hyperbolic Conservation Laws in Continuum Physics; Springer: New York, NY, USA, 2000.

75. Ben-Artzi, M.; Falcovitz, J. Generalized Riemann Problem in Computational Fluid Dynamics; Cambridge University Press: Cambridge, UK, 2003.

76. Conforto F.; Iacono, S.; Oliveri, F. On the generalized Riemann problem for a $2 \times 2$ system of balance laws. In Proceedings "Wascom 2007”, 14th Conference on Waves and Stability in Continuous Media, Scicli (Ragusa), Italy, June 30 - July 6, 2007; Manganaro, N., Monaco, R., Rionero, S., Eds; World Scientific: Singapore, 2008; pp. 162-167.

77. Abraham-Shrauner, B; Govinder, K.S.; Leach, P.G.L. Integration of second order equations not possessing point symmetries. Phys. Lett. A 1995, 203, 169-174.

78. Abraham-Shrauner, B. Hidden symmetries and linearization of the modified Painlevé-Ince equation. J. Math. Phys. 1993, 34, 4809-4816.

79. Abraham-Shrauner, B.; Guo A. Hidden symmetries associated with the projective group of nonlinear first order ordinary differential equations. J. Phys. A-Math. Gen. 1992, 25, 5597-5608.

80. Anco, S.C.; Bluman, G. Derivation of conservation laws from nonlocal symmetries of differential equations. J. Math. Phys. 1996, 37, 2361-2375.

81. Leach, P.G.L.; Maartens, R.; Maharaj, S.D. Self-similar solutions of the generalized Emden-Fowler equation. Int. J. Non-linear Mech. 1992, 27, 575-582. 
82. Muriel, C.; Romero, J.L. New methods of reduction for ordinary differential equations. IMA $J$. Appl. Math. 2001, 66, 111-125.

83. Muriel, C.; Romero, J.L. Prolongations of vector fields and the invariants-by-derivation property. Theor. Math. Phys. 2002, 133, 1565-1575.

84. Muriel, C.; Romero, J.L.; Olver, P.J. Variational $C^{\infty}$-symmetries and Euler-Lagrange equations. $J$. Differ. Equations 2006, 222, 164-184.

85. Cicogna, G.; Gaeta, G.; Morando, P. On the relation between standard and $\mu$-symmetries for PDEs. J. Phys. A-Math. Gen. 2004, 37, 9467-9486.

86. Gaeta, G.; Morando, P. On the geometry of lambda-symmetries and PDEs reduction. J. Phys. A-Math. Gen. 2004, 37, 6955-6975.

87. Catalano Ferraioli, D.; Morando, P. Nonlocal interpretation of $\lambda$-variational symmetry reduction method. 2009, arXiv: 0903.1014v2. Available online: http://arxiv.org/abs/0903.1014 (accessed on November 10, 2009).

88. Krause, J. On the complete symmetry group of the classical Kepler system. J. Math. Phys. 1994, $35,5734-5748$.

89. Andriopoulos, K.; Leach, P.G.L.; Flessas, G.P. Complete symmetry groups of ordinary differential equations and their integrals: Some basic considerations. J. Math. Anal. Appl. 2001, 262, 256-273.

90. Nucci, M.C. The complete Kepler group can be derived by Lie group analysis. J. Math. Phys. 1996, 37, 1772-1775.

91. Myeni, S.M.; Leach, P.G.L. Heuristic analysis of the complete symmetry group and nonlocal symmetries for some nonlinear evolution equations. Math. Meth. Appl. Sci. 2007, 30, 2065-2077.

92. Ibragimov, N.H.; Torrisi, M. Equivalence groups for balance equations. J. Math. Anal. Appl. 1994, 184, 441-452.

93. Ibragimov, N.H.; Torrisi, M.; Valenti, A. Preliminary group classification of equations $v_{t t}=$ $f\left(x, v_{x}\right) v_{x x}+g\left(x, v_{x}\right)$. J. Math. Phys. 1991, 32, 2988-2995.

94. Rosenhaus, V. On one-to-one correspondence between the equation and its group. The Monge-Ampère equation. Preprint F. 18 Acad. Sci. Estonian SSR-Tartu, 1982, 1-16.

95. Rosenhaus, V. The unique determination of the equation by its invariance group and field-space symmetry. Algebras Groups Geom. 1986, 3, 148-166.

96. Rosenhaus, V. Groups of invariance and solutions of equations determined by them. Algebras Groups Geom. 1988, 5, 137-150.

97. Manno, G.; Oliveri, F.; Vitolo, R. On differential equations characterized by their Lie point symmetries. J. Math. Anal. Appl. 2007, 332, 767-786.

98. Manno, G.; Oliveri, F.; Vitolo, R. Differential equations uniquely determined by algebras of point symmetries. Theor. Math. Phys. 2007, 151, 843-850.

99. Manno, G.; Oliveri, F.; Vitolo, R. On the correspondence between differential equations and symmetry algebras. In Symmetry and Perturbation Theory, Proceedings of the International Conference SPT 2007; Gaeta, G., Vitolo, R., Walcher, S., Eds; World Scientific: Singapore, 2007; pp. 164-171. 
(c) 2010 by the author; licensee Molecular Diversity Preservation International, Basel, Switzerland. This article is an open-access article distributed under the terms and conditions of the Creative Commons Attribution license http://creativecommons.org/licenses/by/3.0/. 